\title{
MAXIMIZATION OF THE LONG-TERM GROWTH RATE FOR A PORTFOLIO WITH FIXED AND PROPORTIONAL TRANSACTION COSTS
}

\author{
TAKASHI TAMURA, * Osaka University
}

\begin{abstract}
We study the problem of maximizing the long-run average growth of total wealth for a logarithmic utility function under the existence of fixed and proportional transaction costs. The market model consists of one riskless asset and $d$ risky assets. Impulsive control theory is applied to this problem. We derive a quasivariational inequality (QVI) of 'ergodic' type and obtain a weak solution for the inequality. Using this solution, we obtain an optimal investment strategy to achieve the optimal growth.

Keywords: Portfolio optimization; transaction cost; quasivariational inequality; impulsive control

2000 Mathematics Subject Classification: Primary 91B28

Secondary 93E20; 49N25
\end{abstract}

\section{Introduction}

Merton [9], [10] introduced optimal portfolio management and consumption problems for the Black-Scholes model and obtained the optimal rule. However, he assumed that there were no transaction costs, where the continuous in time rebalancing of the assets was permitted. The optimal strategy in his study required an infinite number of transactions to keep the proportions of the portfolio at a fixed ratio, the so-called Merton ratio. Since then, as one of the more realistic market models, portfolio optimization problems with transaction costs have been widely studied by various researchers.

Taksar et al. [15] studied the problem of maximizing the long-run average growth of total wealth under the existence of proportional transaction costs. Their study was extended by Akian et al. [1] to a multidimensional market model case. Davis and Norman [6] studied the problem of maximizing the expected utility of consumption in the presence of the same transaction costs as [15]. They applied the theory of stochastic singular control to the problem, and performed rigorous analysis and numerical computations. However, in their models [1], [6], [15] a continuous in time rebalancing of the assets is permitted. The optimal strategy involves carrying out infinitesimally small transactions. This does not occur in practice.

Morton and Pliska [11] introduced fixed transaction costs, originally introduced by Duffie and Sun [7], which abandoned carrying out such infinitesimally small transactions. They studied the same maximization problem as [15] in a multidimensional market model and formulated it as an impulsive control problem. Nagai [12] performed a mathematically rigorous analysis of the problem. Although it was to their advantage that the obtained optimal strategy fit practical behaviors better than previous ones, their model did not include the proportional component of transaction costs described above.

Received 12 September 2007; revision received 14 May 2008.

* Postal address: Graduate School of Engineering Science, Osaka University, Toyonaka, 560-8531, Japan.

Email address: tamura@sigmath.es.osaka-u.ac.jp 
In a recent paper [16], not only fixed transaction costs based on [11] but also proportional transaction costs based on [15] for a market model with one riskless asset and one risky asset were introduced. The same problem of maximizing the growth rate as [15] was studied and an optimal investment strategy was obtained.

At the same time and independently, Irle and Sass [8] also studied the exact same problem as [16]. Although the existence of a quasivariational inequality (QVI) and an optimal investment strategy had been proved in [16], they successfully obtained the explicit expression. However, it might not be possible to apply their method to multidimensional cases, because their method relies heavily on the fact that the equation is one-dimensional. In contrast, some methods used in [16] can still be applied to multidimensional cases.

In this paper we extend the result in [16] to a multidimensional market model case with one riskless asset and $d$ risky assets, whose prices are driven by geometric Brownian motions with a constant drift vector and a constant volatility matrix. An investor changes the proportions of the risky assets at each stopping time $\tau_{n}$. The transactions are random variables $\xi_{n}=\left(\xi_{n}^{1}, \ldots, \xi_{n}^{d}\right)$ that are measurable with respect to $\mathcal{F}_{\tau_{n}}$. Fixed and proportional transaction costs must be paid at each trade. The logarithmic utility function is assumed. Borrowing and short selling are not permitted.

Bielecki and Pliska [5, Section 5] studied the same optimization problem as that in this paper under the setting of general proportional transaction costs. They derived an optimal investment strategy by supposing the existence and some properties of the solution to the QVI.

We emphasize two achievements of this study. One is obtaining a weak solution of the QVI and an optimal investment strategy. In contrast, Bielecki and Pliska [5] did not prove the existence of the solution, and Akian et al. [1] required the regularity assumption of a viscosity solution to obtain their optimal strategy. The other achievement of the present paper is the elimination of an assumption that transaction costs must be sufficiently small, which is a required condition in previous studies [12], [16].

This paper is organized in the following manner. In Section 2 we introduce a cost function and rewrite it in a multiplicative form. By translation we can formulate the optimization problem as an ordinary impulsive control problem in the $d$-dimensional simplex $\Delta$. We introduce a diffusion that corresponds to the proportions of the risky assets. This diffusion degenerates at the boundary of $\Delta$. To avoid this degeneracy, we must reformulate the problem as one on the whole Euclidean space $\mathbb{R}^{d}$ by coordinate transformation, as in [12]. Thus, the diffusion is nondegenerate type and the stochastic differential equation (SDE) of the diffusion turns out to be linear.

In Section 3 we consider an optimal stopping problem, which is required to solve the main problem in this paper. Variational inequality (VI) techniques developed by Bensoussan and Lions [3] do not apply directly because the discount factor $\alpha$ of the VI corresponding to the problem is equal to 0 . We prove the existence of the weak solution $\bar{u}$ of the VI by considering the limit of $u_{\alpha}$ as $\alpha \rightarrow 0$ ( $u_{\alpha}$ is the solution of the VI with $\alpha>0$ ). In the proof, it is essential to obtain the upper bound estimate of $u_{\alpha}$ independent of $\alpha$. We obtain the estimate using a property of the coordinate transformation for $\Delta$ and some assumptions. The key assumption is Assumption 3.1, in which any two components of the drift of the log-price process are not equal and none of the components are equal to the risk-free rate.

In Section 4 we obtain a weak solution of the QVI of 'ergodic' type. The pair $(v, l)$ of a function $v$ and a constant $l$ is defined as the solution of the equation. We use perturbation methods studied by Robin [13], [14] to obtain the solution for this type of QVI, in which we consider the limit of $\left(v_{\alpha}-\inf v_{\alpha}, \alpha \inf v_{\alpha}\right)$ as $\alpha \rightarrow 0\left(v_{\alpha}\right.$ is the solution of the QVI with 
$\alpha>0$ ). Robin's results cannot be directly applied to the present problem, because the diffusion process corresponding to the QVI does not have any invariant measure (so we use the notation 'ergodic'). With both perturbation methods and the results in Section 3, we obtain the weak solution of the QVI.

Finally, in Section 5 we attain an optimal investment strategy for the optimization problem by using the results obtained in Sections 3 and 4.

\section{Formulation of portfolio optimization}

Let us consider the following market model, which consists of one riskless asset and $d$ risky assets. The bond price $B_{t}$ is the solution of an ordinary differential equation:

$$
\mathrm{d} B_{t}=r B_{t} \mathrm{~d} t, \quad B_{0}=\bar{B},
$$

where $r$ is a constant interest rate. We denote the price of the $i$ th risky asset by $S_{t}^{i}, 1 \leq i \leq d$. It is the solution of a stochastic differential equation:

$$
\mathrm{d} S_{t}^{i}=S_{t}^{i}\left\{b^{i} \mathrm{~d} t+\sum_{k=1}^{d} \sigma_{k}^{i} \mathrm{~d} W_{t}^{k}\right\}, \quad S_{0}^{i}=s^{i},
$$

where $\boldsymbol{W}_{t}:=\left(W_{t}^{1}, \ldots, W_{t}^{d}\right)^{\top}$ is a $d$-dimensional Brownian motion defined on $\left(\Omega, \mathcal{F}, \mathrm{P}, \mathcal{F}_{t}\right)$, $\boldsymbol{b}:=\left(b^{1}, \ldots, b^{d}\right)^{\top}$ is a constant vector, and $\boldsymbol{\sigma}:=\left(\sigma_{k}^{i}\right)_{i, k=1}^{d}$ is a constant volatility matrix.

Assumption 2.1. We assume that $\sigma \sigma^{\top}$ is positive definite.

We respectively denote by $X_{t}$ and $Y_{t}^{i}$ the amount of money that the investor has in the riskless asset and in the $i$ th risky asset. The total value of the portfolio is $V_{t}=X_{t}+\sum_{i=1}^{d} Y_{t}^{i}$ at time $t$. Our aim is maximizing the asymptotic growth rate under the existence of transaction costs;

$$
\liminf _{T \rightarrow \infty} \frac{1}{T} \mathrm{E}\left[\log V_{T}\right]
$$

where we take the supremum over all the admissible strategies.

\subsection{Definition of transaction costs}

We introduce the following assumption for investment strategies.

Assumption 2.2. We do not allow continuous in time trading. Assets can be traded only at discrete times.

Let $\tau_{n}$ denote the time when the investor trades assets for the $n$th time, and let $\eta_{n}^{i}$ denote the amount of money invested in the $i$ th risky asset at time $\tau_{n}$. Set $\eta_{n}=\left(\eta_{n}^{1}, \ldots, \eta_{n}^{d}\right)^{\top}$. Each investment strategy can be represented as the set of pairs $\left\{\left(\tau_{n}, \boldsymbol{\eta}_{n}\right)\right\}_{n=1}^{\infty}$. Other conditions for the admissibility of strategies are given later. Now we define transaction costs.

Definition 2.1. Take $0 \leq \lambda_{i}, \mu_{i}<1$ and $0<c_{0}<1$. After the $n$th transaction $\left(\tau_{n}, \boldsymbol{\eta}_{n}\right)$, the assets $X_{\tau_{n}}$ and $Y_{\tau_{n}}$ become

$$
\begin{gathered}
X_{\tau_{n}}=\left(1-c_{0}\right)\left(X_{\tau_{n}-}-\sum_{i=1}^{d}\left(\left(1+\lambda_{i}\right) \eta_{n,+}^{i}-\left(1-\mu_{i}\right) \eta_{n,-}^{i}\right)\right), \\
Y_{\tau_{n}}^{i}=\left(1-c_{0}\right)\left(Y_{\tau_{n}-}^{i}+\eta_{n}^{i}\right),
\end{gathered}
$$


where $\eta_{n,+}^{i}$ and $\eta_{n,-}^{i}$ are respectively the positive part and the negative part of $\eta_{n}^{i}$. Here $\eta_{n}^{i}=\eta_{n,+}^{i}-\eta_{n,-}^{i}$ holds.

The fixed components of transaction costs were called 'portfolio management fees' by Duffie and Sun [7, p. 37]. They stated, 'The portfolio management fee is meant to include the cost of adjusting the portfolio and the cost of processing information.' It is the model of aggregate costs, not necessarily money, which prevents investors from trading assets continuously in time.

Assumption 2.3. We do not allow borrowing and short selling.

Hence, $X_{\tau_{n}} \geq 0$ and $Y_{\tau_{n}}^{i} \geq 0$ must hold after each trade. Each $\eta_{n}$ must satisfy

$$
-Y_{\tau_{n}-}^{i} \leq \eta_{n}^{i}, \quad-X_{\tau_{n}-} \leq-\sum_{i=1}^{d}\left(\left(1+\lambda_{i}\right) \eta_{n,+}^{i}-\left(1-\mu_{i}\right) \eta_{n,-}^{i}\right) .
$$

We define admissible investment strategies as follows.

Definition 2.2. The set of pairs $\left\{\left(\tau_{n}, \boldsymbol{\eta}_{n}\right)\right\}_{n=1}^{\infty}$ is said to be an admissible monetary strategy if the following conditions are satisfied.

- $\tau_{n}$ is a stopping time with respect to $\mathcal{F}_{t}$. Here $\left\{\tau_{n}\right\}$ is an increasing sequence, i.e. $\tau_{1}<$ $\tau_{2}<\cdots$.

- $\boldsymbol{\eta}_{n}$ is an $\mathcal{F}_{\tau_{n}}$-measurable random variable and $\mathbb{R}^{d}$-valued.

- $\eta_{n}$ satisfies (2.3).

By the definition of transaction costs we have

$$
\begin{aligned}
V_{\tau_{n}} & =\left(1-c_{0}\right)\left(V_{\tau_{n}-}-\sum_{i=1}^{d}\left(\lambda_{i} \eta_{n,+}^{i}+\mu_{i} \eta_{n,-}^{i}\right)\right) \\
& =\left(1-c_{0}\right)\left(V_{\tau_{n}-}-\tilde{C}\left(\eta_{n}\right)\right),
\end{aligned}
$$

where $\tilde{C}(\boldsymbol{\eta}):=\sum_{i=1}^{d}\left(\lambda_{i} \eta_{+}^{i}+\mu_{i} \eta_{-}^{i}\right)$. We have $0<V_{\tau_{n}-}-\tilde{C}\left(\boldsymbol{\eta}_{n}\right)$ for all the admissible monetary strategies. This means that we do not go bankrupt because of transaction costs without borrowing and short selling.

\subsection{Equivalence of two types of admissible strategies}

When $V_{t} \neq 0$, we define

$$
\boldsymbol{h}_{t}=\left(h_{t}^{1}, \ldots, h_{t}^{d}\right)^{\top}, \quad h_{t}^{i}=\frac{Y_{t}^{i}}{V_{t}} .
$$

Note that $X_{t}=V_{t}\left(1-\sum_{i=1}^{d} h_{t}^{i}\right), h_{t}^{i} \geq 0$, and $\sum_{i=1}^{d} h_{t}^{i} \leq 1$ for all $t \geq 0$. Let $\Delta=\{\boldsymbol{h} \in$ $\left.\mathbb{R}^{d} \mid h^{i} \geq 0, \sum_{i=1}^{d} h_{t}^{i} \leq 1\right\}$. When $V_{t}$ and $\boldsymbol{h}_{t}$ are given, $X_{t}$ and $Y_{t}$ are uniquely determined. We can rewrite (2.3) as

$$
\begin{gathered}
-V_{\tau_{n}} h_{\tau_{n}}^{i} \leq \eta_{n}^{i} \\
-V_{\tau_{n}}\left(1-\sum_{i=1}^{d} h_{\tau_{n}-}^{i}\right) \leq-\sum_{i=1}^{d}\left(\left(1+\lambda_{i}\right) \eta_{n,+}^{i}-\left(1-\mu_{i}\right) \eta_{n,-}^{i}\right) .
\end{gathered}
$$


The main idea to solve the underlying optimization problem is to consider $\left(\boldsymbol{h}_{t}, V_{t}\right)$ as a controllable process instead of $\left(X_{t}, Y_{t}\right)$. For this purpose, we need to rewrite the transaction costs defined in (2.2). Before doing this, we define another type of admissible strategy. Let $\xi_{n}^{i}=h_{\tau_{n}}^{i}-h_{\tau_{n}-}^{i}$, and let $\xi_{n}=\left(\xi_{n}^{1}, \ldots, \xi_{n}^{d}\right)^{\top}$.

Definition 2.3. The set of pairs $\left\{\left(\tau_{n}, \boldsymbol{\xi}_{n}\right)\right\}_{n=1}^{\infty}$ is said to be an admissible proportional strategy for $\boldsymbol{h}_{t}$ if the following conditions are satisfied:

- $\tau_{n}$ satisfies the same conditions as before;

- $\boldsymbol{\xi}_{n}$ is an $\mathcal{F}_{\tau_{n}}$-measurable random variable and $\mathbb{R}^{d}$-valued, and satisfies $\boldsymbol{h}_{\tau_{n}-}+\boldsymbol{\xi}_{n} \in \Delta$.

Monetary strategies determine how much money you invest in each asset. On the other hand, proportional strategies determine each proportion of the assets.

By the definition we have

$$
\xi_{n}^{i}=\frac{Y_{\tau_{n}}^{i}}{V_{\tau_{n}}}-h_{\tau_{n}-}^{i}=\frac{Y_{\tau_{n}-}^{i}+\eta_{n}^{i}}{V_{\tau_{n}-}-\tilde{C}\left(\eta_{n}\right)}-\frac{Y_{\tau_{n}-}^{i}}{V_{\tau_{n}-}} .
$$

From the above we can see that each admissible monetary strategy determines a unique proportional strategy. We define an $\mathbb{R}^{d}$-valued function $\boldsymbol{G}_{V, \boldsymbol{h}}\left(\eta^{1}, \ldots, \eta^{d}\right)$ for $\eta, V-\tilde{C}(\boldsymbol{\eta}) \neq 0$, as follows:

$$
G_{V, \boldsymbol{h}}^{i}(\boldsymbol{\eta}):=\frac{V h^{i}+\eta^{i}}{V-\tilde{C}(\boldsymbol{\eta})}-h^{i} .
$$

By using $\boldsymbol{G}_{V, \boldsymbol{h}}(\cdot)$ we can derive a unique proportional strategy from each monetary strategy, such as $\xi_{n}^{i}=G_{V_{\tau_{n}-}, \boldsymbol{h}_{\tau_{n}-}}^{i}\left(\boldsymbol{\eta}_{n}\right)$.

Here is a question. Is the converse of the above true, i.e. can we uniquely determine a monetary strategy from each proportional strategy? The answer is yes. This result is trivial without transaction costs because trading the $i$ th risky asset does not change the proportions of the other assets $h^{j}, j \neq i$. But it does change $h^{j}$ under the existence of transaction costs through decreasing the total wealth. The following theorem is not trivial.

Theorem 2.1. Let $0 \leq \lambda_{i}, \mu_{i}<1$ and $0<c_{0}<1$. Fix $V>0$. Each admissible proportional strategy $\left\{\left(\tau_{n}, \xi_{n}\right)\right\}_{n=1}^{\infty}$ determines a unique monetary strategy $\left\{\left(\tau_{n}, \eta_{n}\right)\right\}_{n=1}^{\infty}$. Moreover, there exists a Lipschitz continuous function $c(\boldsymbol{h}, \boldsymbol{\xi})$ such that

$$
V_{\tau_{n}}=\left(1-c_{0}\right)\left(1-c\left(\boldsymbol{h}_{\tau_{n}-}, \boldsymbol{\xi}_{n}\right)\right) V_{\tau_{n}-} .
$$

We can rewrite transaction costs (2.4) as above.

Proof. Let us define

$$
\begin{gathered}
A^{\boldsymbol{h}}:=\left\{\boldsymbol{\xi} \in \mathbb{R}^{d} \mid \boldsymbol{h}+\boldsymbol{\xi} \in \Delta\right\}, \\
A_{V, \boldsymbol{h}}:=\left\{\boldsymbol{\eta} \in \mathbb{R}^{d} \mid \boldsymbol{\eta} \text { satisfies (2.5) and (2.6) for } V, \boldsymbol{h}\right\} .
\end{gathered}
$$

We prove that $\boldsymbol{G}_{V, \boldsymbol{h}}(\boldsymbol{\eta})$ is a bijection from $A_{V, \boldsymbol{h}}$ to $A^{\boldsymbol{h}}$ and that there exists a Lipschitz continuous function $c(\boldsymbol{h}, \boldsymbol{\xi})$ such that

$$
\tilde{C}\left(\boldsymbol{G}_{V, \boldsymbol{h}}^{-1}(\boldsymbol{\xi})\right)=c(\boldsymbol{h}, \boldsymbol{\xi}) V
$$

From these results and (2.4), we can obtain the conclusion of the theorem. 
Let $\boldsymbol{\xi}=\boldsymbol{G}_{V, \boldsymbol{h}_{0}}(\boldsymbol{\eta})$ and $\boldsymbol{h}=\boldsymbol{h}_{0}+\boldsymbol{\xi}$. We define a function $\pi: \mathbb{R}^{d} \rightarrow\{-1,0,1\}^{d}$,

$$
\pi^{i}(\eta)= \begin{cases}1 & \text { if } \eta^{i}>0 \\ 0 & \text { if } \eta^{i}=0 \\ -1 & \text { if } \eta^{i}<0\end{cases}
$$

Define the $d \times d$ matrix $\boldsymbol{K}_{\boldsymbol{h}}^{\pi}:=\left(k_{i j}^{\pi}\right)$ by

$$
k_{i j}= \begin{cases}\lambda_{j} h^{i} & \text { if } \pi^{i}=1, \\ 0 & \text { if } \pi^{i}=0, \\ -\mu_{j} h^{i} & \text { if } \pi^{i}=-1 .\end{cases}
$$

By the definition of $\boldsymbol{G}_{V, \boldsymbol{h}_{0}}$ we have

$$
\xi=\boldsymbol{G}_{V, \boldsymbol{h}_{0}}(\boldsymbol{\eta})=\frac{1}{V}\left(\boldsymbol{I}+\boldsymbol{K}_{\boldsymbol{h}}^{\pi(\boldsymbol{\eta})}\right) \boldsymbol{\eta}
$$

where $\boldsymbol{I}$ is an identity matrix. Since $0 \leq \mu_{i}, \lambda_{i}<1$ and $0 \leq \sum_{i=1}^{d} h^{i} \leq 1, \boldsymbol{I}+\boldsymbol{K}_{\boldsymbol{h}}^{\pi}$ is regular for any $\boldsymbol{h}$ and $\pi$. Moreover, if $\left(\boldsymbol{I}+\boldsymbol{K}_{\boldsymbol{h}}^{\pi_{1}}\right) \eta=\left(\boldsymbol{I}+\boldsymbol{K}_{\boldsymbol{h}}^{\pi_{2}}\right) \zeta$ then $\pi_{1}^{i}=\pi_{2}^{i}$ or $\eta^{i}=\zeta^{i}=0$ holds for each $i, 1 \leq i \leq d$. From these results, it is easy to see that $\boldsymbol{G}_{V, \boldsymbol{h}_{0}}(\cdot)$ is an injection.

It follows that

$$
\eta=V\left(\boldsymbol{I}+\boldsymbol{K}_{\boldsymbol{h}}^{\pi(\eta)}\right)^{-1} \boldsymbol{\xi}
$$

By induction in $d$ we can prove that there uniquely exists $\pi_{\xi} \in\{-1,0,1\}^{d}$ for any $\xi$ satisfying $\boldsymbol{h}_{0}+\boldsymbol{\xi} \in \Delta$ such that

$$
\boldsymbol{G}_{V, \boldsymbol{h}_{0}}\left(V\left(\boldsymbol{I}+\boldsymbol{K}_{\boldsymbol{h}_{0}+\boldsymbol{\xi}}^{\pi_{\boldsymbol{\xi}}}\right)^{-1} \boldsymbol{\xi}\right) \equiv \boldsymbol{\xi}, \quad V\left(\boldsymbol{I}+\boldsymbol{K}_{\boldsymbol{h}_{0}+\boldsymbol{\xi}}^{\pi_{\xi}}\right)^{-1} \boldsymbol{\xi} \in A_{V, \boldsymbol{h}_{0}} .
$$

Since the proof is lengthy, we omit it. Hence, $\boldsymbol{G}_{V, \boldsymbol{h}_{0}}(\cdot)$ is a surjection and

$$
\boldsymbol{G}_{V, \boldsymbol{h}_{0}}^{-1}(\boldsymbol{\xi}) \equiv V\left(\boldsymbol{I}+\boldsymbol{K}_{\boldsymbol{h}_{0}+\boldsymbol{\xi}}^{\pi_{\boldsymbol{\xi}}}\right)^{-1} \boldsymbol{\xi}
$$

holds. Moreover, from the definition of $\tilde{C}(\cdot)$ we have

$$
\begin{aligned}
\tilde{C}\left(\boldsymbol{G}_{V, \boldsymbol{h}_{0}}^{-1}(\boldsymbol{\xi})\right) & =\tilde{C}\left(V\left(\boldsymbol{I}+\boldsymbol{K}_{\boldsymbol{h}_{0}+\xi}^{\pi_{\xi}}\right)^{-1} \boldsymbol{\xi}\right) \\
& =V \tilde{C}\left(\left(\boldsymbol{I}+\boldsymbol{K}_{\boldsymbol{h}_{0}+\xi}^{\pi_{\xi}}\right)^{-1} \boldsymbol{\xi}\right) \\
& =c\left(\boldsymbol{h}_{0}, \boldsymbol{\xi}\right) V,
\end{aligned}
$$

where

$$
c\left(\boldsymbol{h}_{0}, \boldsymbol{\xi}\right):=\tilde{C}\left(\left(\boldsymbol{I}+\boldsymbol{K}_{\boldsymbol{h}_{0}+\xi}^{\pi_{\xi}}\right)^{-1} \boldsymbol{\xi}\right) .
$$

We show that $c\left(\boldsymbol{h}_{0}, \boldsymbol{\xi}\right)$ is Lipschitz continuous. Let us use the notation $\pi_{\boldsymbol{h}_{0}, \boldsymbol{\xi}}$ instead of $\pi_{\boldsymbol{\xi}}$ to clarify the dependence. It is easy to see that $\left(\boldsymbol{I}+\boldsymbol{K}_{\boldsymbol{h}_{0}+\xi}^{\pi_{h_{0}, \boldsymbol{\xi}}}\right)^{-1} \boldsymbol{\xi}$ is continuous in $\boldsymbol{h}_{0}$ and $\boldsymbol{\xi}$. It holds that

$$
\min _{\substack{\boldsymbol{\pi} \in\{-1,0,1\}^{d} \\ \boldsymbol{h} \in \Delta}} \operatorname{det}\left(\boldsymbol{I}+\boldsymbol{K}_{\boldsymbol{h}}^{\pi}\right) \neq 0 .
$$


From Cramer's formula, $\left(\boldsymbol{I}+\boldsymbol{K}_{\boldsymbol{h}_{0}+\boldsymbol{\xi}}^{\pi_{0}}\right)^{-1} \boldsymbol{\xi}$ is Lipschitz continuous in $\boldsymbol{h}_{0}$ and $\boldsymbol{\xi}$ for a fixed $\pi_{0}$. Moreover, if $\pi_{1}^{i}=\pi_{2}^{i}, \pi_{1}^{i}=0$, or $\pi_{2}^{i}=0$ holds for each $i, 1 \leq i \leq d$, then we have

$$
\left|\left(\boldsymbol{I}+\boldsymbol{K}_{\boldsymbol{h}_{0}+\xi}^{\pi_{1}}\right)^{-1} \boldsymbol{\xi}-\left(\boldsymbol{I}+\boldsymbol{K}_{f_{0}+v}^{\pi_{2}}\right)^{-1} \boldsymbol{v}\right| \leq L\left(|\xi-\boldsymbol{v}|+\left|\boldsymbol{h}_{0}-\boldsymbol{f}_{0}\right|\right)
$$

for a certain constant $L$. This implies that $\left(\boldsymbol{I}+\boldsymbol{K}_{\boldsymbol{h}_{0}+\xi}^{\pi_{\boldsymbol{h}_{0}, \boldsymbol{\xi}}}\right)^{-1} \boldsymbol{\xi}$ is Lipschitz continuous in each $\xi^{i}$ and $\boldsymbol{h}_{0}^{i}, 1 \leq i \leq d$. With the fact that $\tilde{C}(\cdot)$ is Lipschitz continuous, we obtain the desired result.

Remark 2.1. An elementary calculation shows the triangle inequality for the cost function $c(\cdot, \cdot)$. Under the assumptions of Theorem 2.1 , it holds that

$$
(1-c(\boldsymbol{h}, \boldsymbol{g}-\boldsymbol{h}))(1-c(\boldsymbol{g}, \boldsymbol{f}-\boldsymbol{g})) \leq(1-c(\boldsymbol{h}, \boldsymbol{f}-\boldsymbol{h}))
$$

for any $\boldsymbol{f}, \boldsymbol{g}, \boldsymbol{h} \in \Delta$.

From Theorem 2.1, the underlying optimization problem turns out to be maximizing the growth rate (2.1) over all the admissible proportional strategies. In the following we consider $\left(\boldsymbol{h}_{t}, V_{t}\right)$ as a controllable process.

\subsection{Maximizing the growth of portfolio}

Under the condition of self-financing, we have

$$
\mathrm{d} h_{t}^{i}=h_{t}^{i}\left(\boldsymbol{e}_{i}^{\top}-\boldsymbol{h}_{t}^{\top}\right)\left(\boldsymbol{b}-r \mathbf{1}-\boldsymbol{\sigma} \boldsymbol{\sigma}^{\top} \boldsymbol{h}_{t}\right) \mathrm{d} t+h_{t}^{i}\left(\boldsymbol{e}_{i}^{\top}-\boldsymbol{h}_{t}^{\top}\right) \boldsymbol{\sigma} \mathrm{d} \boldsymbol{W}_{t}
$$

and

$$
\frac{\mathrm{d} V_{t}}{V_{t}}=\sum_{i=0}^{d} h_{t}^{i} \frac{\mathrm{d} S_{t}^{i}}{S_{t}^{i}}=\left(r+\boldsymbol{h}_{t}^{\top}(\boldsymbol{b}-r \mathbf{1})\right) \mathrm{d} t+\boldsymbol{h}_{t}^{\top} \boldsymbol{\sigma} \mathrm{d} \boldsymbol{W}_{t}
$$

for $\tau_{n} \leq t<\tau_{n+1}$, where $\boldsymbol{e}_{i}$ is the $i$ th unit vector and $\mathbf{1}$ is a column vector of $1 \mathrm{~s}$.

Here we see how each admissible proportional strategy $\left\{\left(\tau_{n}, \xi_{n}\right)\right\}_{n=1}^{\infty}$ induces a risky fraction process $h_{t}$. Let $\boldsymbol{h}_{t}^{(0)}$ denote the solution of (2.8) with the initial value $\boldsymbol{h}_{t}^{(0)}=\boldsymbol{h}_{0}$. We define $\boldsymbol{h}_{t}^{(1)}$ as the solution of the following SDE with a random initial condition:

$$
\begin{gathered}
\mathrm{d} h_{t}^{i(1)}=h_{t}^{i(1)}\left(\boldsymbol{e}_{i}^{\top}-\boldsymbol{h}_{t}^{(1)^{\top}}\right)\left(\boldsymbol{b}-r \mathbf{1}-\boldsymbol{\sigma} \boldsymbol{\sigma}^{\top} \boldsymbol{h}_{t}^{(1)}\right) \mathrm{d} t+h_{t}^{i(1)}\left(\boldsymbol{e}_{i}^{\top}-\boldsymbol{h}_{t}^{(1)^{\top}}\right) \boldsymbol{\sigma} \mathrm{d} \boldsymbol{W}_{t}, \quad t \geq \tau_{1}, \\
\boldsymbol{h}_{\tau_{1}}^{(1)}=\boldsymbol{h}_{\tau_{1}}^{(0)}+\boldsymbol{\xi}_{1} .
\end{gathered}
$$

It is known that this SDE has a unique solution (see, e.g. [2, Chapter 1]). By iterating the above definition we can define $\boldsymbol{h}_{t}^{(n)}$ as the solution of the following SDE with a random initial condition:

$$
\begin{gathered}
\mathrm{d} h_{t}^{i(n)}=h_{t}^{i(n)}\left(\boldsymbol{e}_{i}^{\top}-\boldsymbol{h}_{t}^{(n)^{\top}}\right)\left(\boldsymbol{b}-r \mathbf{1}-\boldsymbol{\sigma} \boldsymbol{\sigma}^{\top} \boldsymbol{h}_{t}^{(n)}\right) \mathrm{d} t+h_{t}^{i(n)}\left(\boldsymbol{e}_{i}^{\top}-\boldsymbol{h}_{t}^{(n)^{\top}}\right) \boldsymbol{\sigma} \mathrm{d} \boldsymbol{W}_{t}, \quad t \geq \tau_{n}, \\
\boldsymbol{h}_{\tau_{n}}^{(n)}=\boldsymbol{h}_{\tau_{n}}^{(n-1)}+\xi_{n} .
\end{gathered}
$$

Then we obtain $\boldsymbol{h}_{t}$ defined for each admissible proportional strategy $a_{d m}=\left\{\left(\tau_{n}, \boldsymbol{\xi}_{n}\right)\right\}_{n=1}^{\infty}$ :

$$
\boldsymbol{h}_{t}=\boldsymbol{h}_{t}^{(n)} \quad \text { for } \tau_{n} \leq t<\tau_{n+1} .
$$

The wealth process satisfies

$$
V_{t}\left(1-\boldsymbol{h}_{t}\right)=V_{\tau_{n}}\left(1-\boldsymbol{h}_{\tau_{n}}\right) \exp \left\{r\left(t-\tau_{n}\right)\right\}
$$


during $\tau_{n} \leq t<\tau_{n+1}$. In this paper we consider the problem of maximizing the growth rate of the expected log utility,

$$
J\left(a_{d m}\right):=\liminf _{T \rightarrow \infty} \frac{1}{T} \mathrm{E}\left[\log V_{T}\right]
$$

over all the admissible proportional strategies $\mathcal{A}$. We define

$$
f(\boldsymbol{h}):=-\frac{1}{2} \boldsymbol{h}^{\top} \boldsymbol{\sigma} \boldsymbol{\sigma}^{\top} \boldsymbol{h}+(\boldsymbol{b}-r \mathbf{1})^{\top} \boldsymbol{h}, \quad \boldsymbol{h} \in \mathbb{R}^{d} .
$$

Note that $f(\boldsymbol{h})$ attains the maximum at $\left(\boldsymbol{\sigma} \boldsymbol{\sigma}^{\top}\right)^{-1}(\boldsymbol{b}-r \mathbf{1})$. Let $C(\boldsymbol{h}, \boldsymbol{\xi})=\log \left(1-c_{0}\right)+\log (1-$ $c(\boldsymbol{h}, \boldsymbol{\xi}))$. From (2.7), it holds that

$$
0>C(\boldsymbol{h}, \boldsymbol{f}-\boldsymbol{h}) \geq C(\boldsymbol{h}, \boldsymbol{g}-\boldsymbol{h})+C(\boldsymbol{g}, \boldsymbol{f}-\boldsymbol{g})+\left|\log \left(1-c_{0}\right)\right| .
$$

By applying Itô's formula to $\log V_{t}$ we obtain, from Theorem 2.1,

$$
\begin{aligned}
\log V_{t} & =\log V_{\tau_{n}}+\log \left(1-\boldsymbol{h}_{\tau_{n}}\right)-\log \left(1-\boldsymbol{h}_{t}\right)+r\left(t-\tau_{n}\right) \\
& =\int_{\tau_{n}}^{t}\left(f\left(\boldsymbol{h}_{s}\right)+r\right) \mathrm{d} s+\log V_{\tau_{n}}+\int_{\tau_{n}}^{t} \boldsymbol{h}_{s} \boldsymbol{\sigma} \mathrm{d} W_{s} \\
& =\int_{0}^{t}\left(f\left(\boldsymbol{h}_{s}\right)+r\right) \mathrm{d} s+\log V_{0}+\sum_{k=1}^{n} C\left(\boldsymbol{h}_{\tau_{k}-}, \boldsymbol{\xi}_{k}\right)+\int_{0}^{t} \boldsymbol{h}_{s} \boldsymbol{\sigma} \mathrm{d} W_{s}
\end{aligned}
$$

for $\tau_{n-1} \leq t<\tau_{n}$. So we obtain

$$
J\left(a_{d m}\right)=r+\liminf _{T \rightarrow \infty} \frac{1}{T} \mathrm{E}\left[\int_{0}^{T} f\left(\boldsymbol{h}_{s}\right) \mathrm{d} s+\sum_{k=1}^{\infty} C\left(\boldsymbol{h}_{\tau_{k}-}, \boldsymbol{\xi}_{k}\right) 1_{\left\{\tau_{k}<T\right\}}\right]
$$

where $1_{\{.\}}$denotes the indicator function. We shall obtain the optimal growth rate

$$
\rho:=\sup _{a_{d m} \in \mathcal{A}} J\left(a_{d m}\right)
$$

and the optimal strategy $\hat{a}_{d m}=\left\{\left(\hat{\tau}_{n}, \hat{\boldsymbol{\xi}}_{n}\right)\right\}$ that attains the optimal growth rate. Let us define

$$
l^{*}:=\rho-r
$$

Although $\boldsymbol{h}_{t}$ has a clear meaning and $f(\boldsymbol{h})$ is simple, it is difficult to treat $\boldsymbol{h}_{t}$ directly. This is because $\boldsymbol{h}_{t}$ is degenerate at the boundary of $\Delta$. So we apply a coordinate transformation to $\boldsymbol{h}_{t}$ and obtain another representation of (2.11). We define a coordinate transformation $\psi$ as

$$
y^{i}=\psi^{i}\left(h^{1}, h^{2}, \ldots, h^{d}\right)=\log h^{i}-\log \left(1-\sum_{j=1}^{d} h^{j}\right) .
$$

Then the inverse mapping $\phi:=\psi^{-1}$ can be defined by

$$
h^{i}=\phi\left(y^{1}, y^{2}, \ldots, y^{d}\right)=\frac{\exp \left\{y^{i}\right\}}{1+\sum_{j=1}^{d} \exp \left\{y^{j}\right\}} .
$$


Let $\boldsymbol{y}_{t}=\psi\left(\boldsymbol{h}_{t}\right)$. It holds that

$$
y_{t}^{i}=\log h_{t}^{i}-\log \left(1-\sum_{j=1}^{d} h_{t}^{j}\right)=\log \frac{Y_{t}^{i}}{V_{t}}-\log \frac{X_{t}}{V_{t}}=\log \frac{Y_{t}^{i}}{X_{t}} .
$$

Therefore, $\boldsymbol{y}_{t}$ is governed by the stochastic differential equation

$$
\mathrm{d} y_{t}^{i}=\left(b^{i}-r-\frac{1}{2}\left(\boldsymbol{\sigma} \boldsymbol{\sigma}^{\top}\right)^{i i}\right) \mathrm{d} t+\left(\boldsymbol{\sigma} \mathrm{d} \boldsymbol{W}_{t}\right)^{i} \quad \text { for } \tau_{n-1} \leq t<\tau_{n},
$$

where $y_{t}^{i}$ is the logarithm of the discounted $i$ th asset price process. Note that

$$
f\left(\boldsymbol{e}_{i}\right)=\boldsymbol{f}(0, \ldots, 1, \ldots, 0)=b^{i}-r-\frac{1}{2}\left(\boldsymbol{\sigma} \boldsymbol{\sigma}^{\top}\right)^{i i} .
$$

This is not a coincidence. If we buy only the $i$ th risky asset and continue to hold it without trading, then we attain the growth rate $\lim \inf \mathrm{E}\left[\log V_{T}\right] / T=f\left(\boldsymbol{e}_{i}\right)+r$. This is why $f\left(\boldsymbol{e}_{i}\right)$ coincides with the drift of $y_{t}^{i}$. Let us define

$$
\bar{f}(\boldsymbol{y}):=f(\phi(y)), \quad \bar{C}(\boldsymbol{y}, \zeta):=C(\phi(y), \phi(y+\zeta)-\phi(y)) .
$$

We can rewrite (2.11) and (2.12) as

$$
\begin{gathered}
\tilde{J}\left(\tilde{a}_{d m}\right):=r+\liminf _{T \rightarrow \infty} \frac{1}{T} \mathrm{E}\left[\int_{0}^{T} \bar{f}\left(\boldsymbol{y}_{s}\right) \mathrm{d} s+\sum_{k=1}^{\infty} \bar{C}\left(\boldsymbol{y}_{\tau_{k}-}, \zeta_{k}\right) 1_{\left\{\tau_{k}<T\right\}}\right], \\
\tilde{\rho}:=\sup _{\tilde{a}_{d m} \in \tilde{\mathcal{A}}} \tilde{J}\left(\tilde{a}_{d m}\right),
\end{gathered}
$$

where

$\tilde{\mathcal{A}}:=\left\{\left\{\left(\tau_{n}, \zeta_{n}\right)\right\}_{n=1}^{\infty} \mid \tau_{n}\right.$ satisfies the same conditions as before,

$$
\left.\boldsymbol{y}_{\tau_{n}}+\zeta_{n} \in \mathbb{R}^{d} \text { and } \zeta_{n} \text { is } \mathcal{F}_{\tau_{n}} \text {-measurable }\right\}
$$

In what follows we consider the problem of maximizing $\tilde{J}\left(\tilde{a}_{d m}\right)$ in (2.3) over all the admissible strategies $\tilde{\mathcal{A}}$ and find an optimal strategy $\left\{\left(\hat{\tau}_{n}, \hat{\zeta}_{n}\right)\right\}_{n=1}^{\infty}$ that attains $\tilde{\rho}$. We define $\boldsymbol{y}_{t}$ induced by an admissible strategy $\tilde{a}_{d m} \in \tilde{\mathcal{A}}$ in the same way as $\boldsymbol{h}_{t}$. We must emphasize that if $h_{\tau_{n}-}^{i}+\xi_{n}^{i}=0$ then

$$
\begin{aligned}
\zeta_{n}^{i} & =\psi^{i}\left(\boldsymbol{h}_{\tau_{n}-}+\boldsymbol{\xi}_{n}\right)-\psi^{i}\left(\boldsymbol{h}_{\tau_{n}-}\right) \\
& =\log 0-\log \left(1-\sum_{j=1}^{d}\left(h_{\tau_{n}-}^{j}+\xi_{n}^{j}\right)\right)-\psi^{i}\left(\boldsymbol{h}_{\tau_{n}-}\right)
\end{aligned}
$$

$\zeta_{n}$ cannot be admissible. So $\tilde{\rho} \leq \rho$. But, in fact, $\tilde{\rho}=\rho$ holds. Let us consider a certain portfolio $\left(\boldsymbol{h}_{t}, V_{t}\right)$ induced by a certain admissible strategy $a_{0} \in \mathcal{A}$ with the initial value $\left(\boldsymbol{h}_{0}, V_{0}\right)$. We can assume that $\boldsymbol{h}_{0} \in \Delta \backslash \partial \Delta$ without loss of generality. At the same time, we consider another portfolio $\left(\boldsymbol{h}_{t}^{\delta}, V_{t}^{\delta}\right)$ with the initial value $\left(\boldsymbol{h}_{0}, V_{0}+\delta\right), \delta>0$, which consists of two parts: one is induced by the strategy $a_{0}$ with the initial wealth $\left(\boldsymbol{h}_{0}, V_{0}\right)$, the other is induced by the strategy of holding the initial wealth $\left(\boldsymbol{h}_{0}, \delta\right)$ without trading. Then a strategy inducing this portfolio is 
included in $\tilde{\mathcal{A}}$ because $\boldsymbol{h}_{t}^{\delta}$ never reaches the boundary $\partial \Delta$. Stock prices never become negative. We obtain

$$
\sup _{a \in \tilde{A}} \lim \inf \frac{1}{T} \mathrm{E}\left[\log V_{T}\right] \leq \sup _{a \in \mathcal{A}} \lim \inf \frac{1}{T} \mathrm{E}\left[\log V_{T}\right] \leq \sup _{a \in \tilde{\mathcal{A}}} \lim \inf \frac{1}{T} \mathrm{E}\left[\log V_{T}^{\delta}\right] .
$$

Since both sides of the above equation do not depend on the initial wealth, the desired result follows.

Proposition 2.1. Under the above settings, it holds that

$$
\rho=\tilde{\rho} .
$$

\section{Optimal stopping problem with no discount factor}

Let $\boldsymbol{y}_{t}$ be the solution of the SDE (2.15) with the initial value $\boldsymbol{y}_{0}=\boldsymbol{y}$. In this section we study a certain type of optimal stopping problem, which is needed to solve the main problem in this paper:

$$
w_{l}(\boldsymbol{y}):=\sup _{\tau} \mathrm{E}_{\boldsymbol{y}}\left[\liminf _{T \rightarrow \infty} \int_{0}^{\tau \wedge T}\left(\bar{f}\left(\boldsymbol{y}_{S}\right)-l\right) \mathrm{d} s+\Psi\left(\boldsymbol{y}_{\tau \wedge T}\right)\right],
$$

where $\tau$ is a stopping time with respect to $\mathscr{F}_{t}$ and $\Psi(\boldsymbol{y})$ is a Lipschitz continuous bounded function on $\mathbb{R}^{d}$. Note that there does not exist any discount factor for this problem. By setting $\tau \equiv 0$ we can see that (3.1) has a trivial lower bound $w_{l}(\boldsymbol{y}) \geq \Psi(\boldsymbol{y})$. Later in this section we remove the lim inf in (3.1), but for now it is needed because $\int_{0}^{\tau}\left(\bar{f}\left(\boldsymbol{y}_{s}\right)-l\right) \mathrm{d} s+\Psi\left(\boldsymbol{y}_{\tau}\right)$ is not well defined on $\{\tau=\infty\}$. Let us define

$$
\beta^{i}:=b^{i}-r-\frac{1}{2}\left(\sigma \sigma^{\top}\right)^{i i}, \quad \bar{\beta}:=\max \beta^{i} \vee 0 .
$$

Assumption 3.1. In the rest of this paper we assume that, for any $i, j$,

$$
\beta^{i} \neq 0, \quad \beta^{i} \neq \beta^{j}
$$

From the definition of $\bar{f}(\cdot)$, it is easy to obtain the following lemma.

Lemma 3.1. Fix any $l>\bar{\beta}$. It holds that

$$
\operatorname{supp}\left[(\bar{f}(\boldsymbol{y})-l)_{+}\right] \subset \bigcup_{i=1}^{d} D_{i} \cup \bigcup_{i, j=1}^{d} D_{i j}
$$

where $D_{i}=\left\{\boldsymbol{y} \in \mathbb{R}^{d}|| y^{i} \mid<C\right\}, D_{i j}=\left\{\boldsymbol{y} \in \mathbb{R}^{d}|| y^{i}-y^{j} \mid<C\right\}, C$ is a certain constant that depends on $l$, and $f_{+}$is the positive part of $f$.

Now we obtain the boundedness of $w_{l}$. It is essential for this paper.

Lemma 3.2. Under assumption (3.2), it holds that

$$
w_{l}(\boldsymbol{y}) \leq \sup _{\boldsymbol{y} \in \mathbb{R}^{d}} \mathrm{E}_{\boldsymbol{y}}\left[\int_{0}^{\infty}\left(\bar{f}\left(\boldsymbol{y}_{t}\right)-l\right)_{+} \mathrm{d} t\right]+\|\Psi\|_{\infty}<\infty \text { for any } l>\bar{\beta} .
$$


Proof. From assumption (3.2), $y_{t}^{i}$ and $y_{t}^{i}-y_{t}^{j}$ are transient for each $i, j$. Hence, Lemma 3.1 implies that

$$
\sup _{\boldsymbol{y} \in \mathbb{R}^{d}} \mathrm{E}_{\boldsymbol{y}}\left[\int_{0}^{\infty} 1_{\mathcal{O}}\left(\boldsymbol{y}_{t}\right) \mathrm{d} t\right]<\infty
$$

for each $\mathcal{O}=D_{i}, D_{i j}$. Since $\bar{f}$ is bounded on $\mathbb{R}^{d}$,

$$
\mathrm{E}\left[\int_{0}^{\infty}\left(\bar{f}\left(\boldsymbol{y}_{t}\right)-l\right)_{+} \mathrm{d} t\right] \leq\|\bar{f}-l\|_{\infty} \sum_{\mathcal{O}=D_{i}, D_{i j}} \sup _{\boldsymbol{y} \in \mathbb{R}^{d}} \mathrm{E}_{\boldsymbol{y}}\left[\int_{0}^{\infty} 1_{\mathcal{O}}\left(\boldsymbol{y}_{t}\right) \mathrm{d} t\right]<\infty .
$$

This completes the proof.

Lemma 3.3. Assume that (3.2) holds. Fix any $l>\bar{\beta}$. If $\mathrm{E}[\tau]=\infty$ then

$$
\mathrm{E}_{\boldsymbol{y}}\left[\liminf _{T \rightarrow \infty} \int_{0}^{\tau \wedge T}\left(\bar{f}\left(\boldsymbol{y}_{S}\right)-l\right) \mathrm{d} s+\Psi\left(\boldsymbol{y}_{\tau \wedge T}\right)\right]=-\infty .
$$

Proof. Take $\delta$ such that $l-\delta>\bar{\beta}$. Then

$$
\int_{0}^{\tau \wedge T}\left(\bar{f}\left(\boldsymbol{y}_{s}\right)-l\right) \mathrm{d} s+\Psi\left(\boldsymbol{y}_{\tau \wedge T}\right) \leq \int_{0}^{\infty}\left(\bar{f}\left(\boldsymbol{y}_{S}\right)-(l-\delta)\right)_{+} \mathrm{d} s+\|\Psi\|_{\infty}-(\tau \wedge T) \delta .
$$

By taking the lim inf and the expectation of both sides, we obtain the result from Lemma 3.2.

So it is enough to consider only the case in which $\tau<\infty$ almost surely (a.s.) for the optimal stopping problem (3.1). Moreover, we have obtained

$$
w_{l}(\boldsymbol{y})=\sup _{\mathrm{E}[\tau]<\infty} \mathrm{E}_{\boldsymbol{y}}\left[\int_{0}^{\tau}\left(\bar{f}\left(\boldsymbol{y}_{s}\right)-l\right) \mathrm{d} s+\Psi\left(\boldsymbol{y}_{\tau}\right)\right] \text { for } l>\bar{\beta} .
$$

The lemma below also follows.

Lemma 3.4. Assume that (3.2) holds. Fix any $l>\bar{\beta}$. Let $\tau$ and $\tau^{\prime}$ be two stopping times with $\tau \leq \tau^{\prime}$. Assume that $\mathrm{E}[\tau]<\infty$. If there exists a constant $K_{\boldsymbol{y}}$ independently of $T$ such that

$$
-K_{\boldsymbol{y}}<\mathrm{E}_{\boldsymbol{y}}\left[\int_{\tau \wedge T}^{\tau^{\prime} \wedge T}\left(\bar{f}\left(\boldsymbol{y}_{S}\right)-l\right) \mathrm{d} s+\Psi\left(\boldsymbol{y}_{\tau^{\prime} \wedge T}\right)\right],
$$

then $\mathrm{E}_{\boldsymbol{y}}\left[\tau^{\prime}\right]<\infty$. If $K_{\boldsymbol{y}}$ is independent of $\boldsymbol{y}$ then $\sup _{\boldsymbol{y} \in \mathbb{R}^{d}} \mathrm{E}_{\boldsymbol{y}}\left[\tau^{\prime}\right]<\infty$.

Proof. In a similar way to (3.3) we obtain

$$
\mathrm{E}_{\boldsymbol{y}}\left[\tau^{\prime} \wedge T\right] \leq \frac{1}{\delta}\left(\mathrm{E}_{\boldsymbol{y}}\left[\int_{0}^{\infty}\left(\bar{f}\left(\boldsymbol{y}_{s}\right)-(l-\delta)\right)_{+} \mathrm{d} s\right]+\|\Psi\|_{\infty}+K_{\boldsymbol{y}}\right)+\mathrm{E}[\tau \wedge T] .
$$

The first result follows from the monotone convergence theorem. Since it holds that

$$
\sup _{\boldsymbol{y} \in \mathbb{R}^{d}} \mathrm{E}_{\boldsymbol{y}}\left[\int_{0}^{\infty}\left(\bar{f}\left(\boldsymbol{y}_{s}\right)-(l-\delta)\right)_{+} \mathrm{d} s\right]<\infty,
$$

we obtain the second result. 


\subsection{Study of the VI and deriving an optimal stopping time}

Let $L$ be the generator of $\boldsymbol{y}_{t}$ :

$$
L:=\frac{1}{2} \sum_{i, j=1}^{d}\left(\sigma \sigma^{\top}\right)^{i j} \frac{\partial^{2}}{\partial x_{i} \partial x_{j}}+\sum_{i=1}^{d}\left(b^{i}-r-\left(\sigma \sigma^{\top}\right)^{i i}\right) \frac{\partial}{\partial x_{i}} .
$$

We consider the following VI:

$$
\max \{L u+\bar{f}-l, \Psi-u\}=0
$$

to solve the optimal stopping problem (3.1), as in [12]. Note that this VI does not have any discount factor. So, we cannot directly apply theorems obtained by Bensoussan and Lions [3]. We define

$$
\begin{array}{rlrl}
m_{\gamma}(\boldsymbol{y}) & :=\exp \left\{-\gamma\left(1+\boldsymbol{y}^{\top}\left(\boldsymbol{\sigma} \boldsymbol{\sigma}^{\top}\right)^{-1} \boldsymbol{y}\right)^{1 / 2}\right\}, & L^{p, \gamma}:=\left\{\left.z\left|\int\right| z\right|^{p} m_{\gamma}^{2} \mathrm{~d} \boldsymbol{y}<\infty\right\}, \\
W^{1, p, \gamma}:=\left\{z \in L^{p, \gamma} \mid D_{i} z \in L^{p, \gamma}\right\}, & W^{2, p, \gamma}:=\left\{z \in W^{1, p, \gamma} \mid D_{i j} z \in L^{p, \gamma}\right\} .
\end{array}
$$

Let $\varepsilon_{\alpha}(u, v)$ be a bilinear form defined on $W^{1,2, \gamma}$ by

$$
\begin{aligned}
\mathcal{E}_{\alpha}(u, v):= & \frac{1}{2} \int(\nabla u)^{\top} \boldsymbol{\sigma} \boldsymbol{\sigma}^{\top}\left(\nabla v-\frac{2 \gamma\left(\boldsymbol{\sigma} \boldsymbol{\sigma}^{\top}\right)^{-1} \boldsymbol{y}}{\sqrt{1+\boldsymbol{y}^{\top}\left(\boldsymbol{\sigma} \boldsymbol{\sigma}^{\top}\right)^{-1} \boldsymbol{y}}}\right) m_{\gamma}^{2} \mathrm{~d} \boldsymbol{y} \\
& +\int \boldsymbol{b}^{\top} \nabla u v m_{\gamma}^{2} \mathrm{~d} \boldsymbol{y}+\alpha \int u v m_{\gamma}^{2} \mathrm{~d} \boldsymbol{y} .
\end{aligned}
$$

It is easy to see that there exists a constant $\lambda_{0}>0$ such that

$$
\varepsilon_{\alpha}(u, u)+\lambda_{0}\|u\|_{L^{2, \gamma}}^{2} \geq k_{0}\|u\|_{W^{1,2, \gamma}}^{2} \quad \text { for } \alpha \geq 0 .
$$

Let us consider (3.4) in a weak sense:

$$
\varepsilon_{0}(u, v-u) \geq(\bar{f}-l, v-u), \quad u \geq \Psi
$$

for all $v \geq \Psi$ and $v \in W^{1,2, \gamma}\left(\mathbb{R}^{d}\right)$. There is a result obtained in [3] for variational inequalities with a discount factor; see Theorem 1.13 of [3].

Theorem 3.1. ([3].) Let $\alpha>0$. Suppose that $\Psi(y)$ is uniformly continuous and bounded. Then there exists a unique continuous solution $u_{\alpha} \in W^{1,2, \gamma}\left(\mathbb{R}^{d}\right)$ of the VI

$$
\varepsilon_{\alpha}\left(u_{\alpha}, v-u_{\alpha}\right) \geq\left(\bar{f}-l, v-u_{\alpha}\right), \quad u_{\alpha} \geq \Psi,
$$

for all $v \geq \Psi$ and $v \in W^{1,2, \gamma}\left(\mathbb{R}^{d}\right)$. Furthermore, $\tau^{*}:=\inf \left\{t>0 \mid \Psi\left(\boldsymbol{y}_{t}\right) \geq u_{\alpha}\left(\boldsymbol{y}_{t}\right)\right\}$ is an optimal stopping time for

$$
\begin{aligned}
u_{\alpha}(\boldsymbol{y}) & =\sup _{\tau} \mathrm{E}\left[\int_{0}^{\tau} \mathrm{e}^{-\alpha s}\left(\bar{f}\left(\boldsymbol{y}_{s}\right)-l\right) \mathrm{d} s+\mathrm{e}^{-\alpha \tau} \Psi\left(\boldsymbol{y}_{\tau}\right)\right] \\
& =\mathrm{E}_{\boldsymbol{y}}\left[\int_{0}^{\tau^{*}} \mathrm{e}^{-\alpha s}\left(\bar{f}\left(\boldsymbol{y}_{s}\right)-l\right) \mathrm{d} s+\mathrm{e}^{-\alpha \tau^{*}} \Psi\left(\boldsymbol{y}_{\tau^{*}}\right)\right] .
\end{aligned}
$$


Let us consider the following penalized equation:

$$
(\alpha-\bar{L}) u_{\alpha}^{\varepsilon}-\frac{1}{\varepsilon}\left(u_{\alpha}^{\varepsilon}-\Psi\right)_{-}=\bar{f}-l
$$

which has a continuous solution $u_{\alpha}^{\varepsilon} \in W^{2, p, \gamma}$. Note that $u_{\alpha}^{\varepsilon}$ has the probabilistic representation

$$
u_{\alpha}^{\varepsilon}(\boldsymbol{y})=\sup _{\gamma} \mathrm{E}_{\boldsymbol{y}}\left[\int_{0}^{\infty} \exp \left\{-\alpha t-\int_{0}^{t} \gamma_{s} \mathrm{~d} s\right\}\left(\bar{f}\left(\boldsymbol{y}_{t}\right)-l+\gamma_{t} \Psi\left(\boldsymbol{y}_{t}\right)\right) \mathrm{d} t\right],
$$

where $\gamma_{t}$ ranges over the set of all the progressively measurable processes such that $0 \leq \gamma_{t} \leq$ $1 / \varepsilon$. Moreover, we have the following theorem. See Theorem 3.7 of [3].

Theorem 3.2. ([3].) Under the assumptions of Theorem 3.1, it follows that

$$
u_{\alpha}^{\varepsilon} \leq u_{\alpha}, \quad u_{\alpha}^{\varepsilon} \rightarrow u_{\alpha} \quad \text { in } C^{0}\left(\mathbb{R}^{d}\right) \text { as } \varepsilon \rightarrow 0 .
$$

Let $\tau_{\alpha}^{*}$ be the optimal stopping time defined in Theorem 3.1 with respect to the discount factor $\alpha$. We can obtain a certain estimate of $\tau_{\alpha}^{*}$ as follows.

Lemma 3.5. Assume that $l>\bar{\beta}$. Then there exist $\alpha_{0}>0$ and $K$ such that, for all $\alpha<\alpha_{0}$,

$$
\mathrm{E}\left[1-\exp \left\{-\alpha \tau_{\alpha}^{*}\right\}\right]<\alpha K .
$$

Here $K$ does not depend on $\alpha$.

Proof. From (3.8) and Lemma 3.2, we obtain

$$
-\|\Psi\|_{\infty} \leq u_{\alpha}(\boldsymbol{y}) \leq \sup _{\boldsymbol{y} \in \mathbb{R}^{d}} \mathrm{E}_{\boldsymbol{y}}\left[\int_{0}^{\infty}\left(f\left(\boldsymbol{y}_{S}\right)-l\right)_{+} \mathrm{d} s\right]+\|\Psi\|_{\infty}<\infty .
$$

In particular, $\left\|u_{\alpha}\right\|_{\infty}<\infty$. Take $\delta>0$ such that $l-\delta>\bar{\beta}$. From Lemma 3.2 we have

$$
\begin{aligned}
\mathrm{E}_{\boldsymbol{y}}\left[\int_{0}^{\tau_{\alpha}^{*}} \mathrm{e}^{-\alpha s}\left(f\left(\boldsymbol{y}_{s}\right)-l\right) \mathrm{d} s\right] & \leq \mathrm{E}_{\boldsymbol{y}}\left[\int_{0}^{\infty}\left(f\left(\boldsymbol{y}_{s}\right)-l+\delta\right)_{+} \mathrm{d} s\right]-\delta \mathrm{E}_{\boldsymbol{y}}\left[\int_{0}^{\tau_{\alpha}^{*}} \mathrm{e}^{-\alpha s} \mathrm{~d} s\right] \\
& <K_{0}-\frac{\delta}{\alpha} \mathrm{E}_{\boldsymbol{y}}\left[1-\exp \left\{-\alpha \tau_{\alpha}^{*}\right\}\right] .
\end{aligned}
$$

From (3.8) and the above, we obtain

$$
\frac{\delta}{\alpha} \mathrm{E}_{y}\left[1-\exp \left\{-\alpha \tau_{\alpha}^{*}\right\}\right] \leq K_{0}+2\left\|u_{\alpha}\right\|_{\infty}
$$

This completes the proof.

We can obtain the existence of the solution of the VI (3.6) as follows. We have not been able to prove the uniqueness of the solution as yet.

Theorem 3.3. Assume that (3.2) holds and that $l>\bar{\beta}$. Assume that $\Psi(\boldsymbol{y})$ is Lipschitz continuous and bounded. Then there exists a bounded continuous solution $\bar{u} \in W^{1, p, \gamma}\left(\mathbb{R}^{d}\right)$ of (3.6). 
Proof. Let $\boldsymbol{y}_{t}$ and $\boldsymbol{y}_{t}^{\prime}$ be solutions of the SDE (2.15) with initial values $\boldsymbol{y}$ and $\boldsymbol{y}^{\prime}$, respectively. Since $\bar{f}$ and $\Psi$ are Lipschitz continuous, we have

$$
\begin{aligned}
u_{\alpha}(\boldsymbol{y})-u_{\alpha}\left(\boldsymbol{y}^{\prime}\right) & \leq \mathrm{E}_{\boldsymbol{y}}\left[\int_{0}^{\tau_{\alpha}^{*}} \mathrm{e}^{-\alpha t}\left(\bar{f}\left(\boldsymbol{y}_{t}\right)-\bar{f}\left(\boldsymbol{y}_{t}^{\prime}\right)\right) \mathrm{d} t\right]+K_{1}\left|\boldsymbol{y}-\boldsymbol{y}^{\prime}\right| \\
& \leq K_{0}\left|\boldsymbol{y}-\boldsymbol{y}^{\prime}\right| \frac{1}{\alpha} \mathrm{E}\left[1-\exp \left\{-\alpha \tau_{\alpha}^{*}\right\}\right]+K_{1}\left|\boldsymbol{y}-\boldsymbol{y}^{\prime}\right| \\
& \leq K_{0} K\left|\boldsymbol{y}-\boldsymbol{y}^{\prime}\right|+K_{1}\left|\boldsymbol{y}-\boldsymbol{y}^{\prime}\right|
\end{aligned}
$$

from Lemma 3.5. By interchanging $\boldsymbol{y}$ and $\boldsymbol{y}^{\prime}$, we obtain

$$
\left|u_{\alpha}\left(\boldsymbol{y}^{\prime}\right)-u_{\alpha}(\boldsymbol{y})\right| \leq K_{2}\left|\boldsymbol{y}-\boldsymbol{y}^{\prime}\right|
$$

From (3.9) and (3.10), it follows that $\left\|u_{\alpha}\right\|_{W^{1, \infty, \gamma}}<C$, where $C$ does not depend on $\alpha$. Hence, there exists a subsequence $u_{\alpha_{k}}$ that weakly converges to a certain bounded function $\bar{u} \in W^{1, p, \gamma}$ as $\alpha_{k} \rightarrow 0$. Furthermore, $u_{\alpha_{k}}$ strongly converges to $\bar{u}$ in $L^{p, \gamma}$ on any bounded domain. Take a domain $D$ such that $\int_{D^{c}} m_{\gamma}^{2} \mathrm{~d} \boldsymbol{y}<\varepsilon$. By sending $\alpha_{k} \rightarrow 0$ we obtain

$$
\begin{aligned}
\int_{\mathbb{R}^{d}}\left|u_{\alpha_{k}}-\bar{u}\right|^{p} m_{\gamma}^{2} \mathrm{~d} \boldsymbol{y} & \leq \int_{D}\left|u_{\alpha_{k}}-\bar{u}\right|^{p} m_{\gamma}^{2} \mathrm{~d} \boldsymbol{y}+\varepsilon\left\|u_{\alpha_{k}}-\bar{u}\right\|_{\infty}^{p} \\
& \leq \varepsilon+\varepsilon C^{p} .
\end{aligned}
$$

We can take $\varepsilon$ arbitrarily. Hence, $u_{\alpha_{k}}$ strongly converges to $\bar{u}$ in $L^{p, \gamma}\left(\mathbb{R}^{d}\right)$.

From (3.7) we have, for all $v \in W^{1,2, \gamma}$ and $v \geq \Psi$,

$$
\varepsilon_{\alpha_{k}}\left(u_{\alpha_{k}}, v\right)-\left(\bar{f}-l, v-u_{\alpha_{k}}\right) \geq \varepsilon_{\alpha_{k}}\left(u_{\alpha_{k}}, u_{\alpha_{k}}\right) .
$$

From the coercivity, (3.5), of $\varepsilon_{\alpha}$, it follows that

$$
\varepsilon_{\alpha_{k}}\left(u_{\alpha_{k}}-\bar{u}, u_{\alpha_{k}}-\bar{u}\right)+\lambda_{0}\left\|u_{\alpha_{k}}-\bar{u}\right\|_{L^{2, \gamma}}^{2} \geq 0 .
$$

By taking the lim inf of the left-hand side we obtain

$$
\liminf _{\alpha_{k} \rightarrow 0} \mathcal{E}_{\alpha_{k}}\left(u_{\alpha_{k}}, u_{\alpha_{k}}\right)-\varepsilon_{0}(\bar{u}, \bar{u}) \geq 0
$$

and

$$
\varepsilon_{0}(\bar{u}, v)-(\bar{f}-l, v-\bar{u}) \geq \liminf \varepsilon_{\alpha_{k}}\left(u_{\alpha_{k}}, u_{\alpha_{k}}\right) \geq \varepsilon_{0}(\bar{u}, \bar{u}) .
$$

This completes the proof.

We can obtain the regularity of the solution $\bar{u}$ if $\Psi$ has enough regularity.

Lemma 3.6. Suppose that the assumptions of Theorem 3.3 hold and that $L \Psi \in L^{p, \gamma}\left(\mathbb{R}^{d}\right)$. Then we have a solution $\bar{u} \in W^{2, p, \gamma}\left(\mathbb{R}^{d}\right)$ of (3.6).

Proof. In the same way as in the proof of Lemma 5.1 of [12], we can show that

$$
-\frac{1}{1+\varepsilon \alpha} K_{0}-\|\Psi\|_{\infty} \leq u_{\alpha}^{\varepsilon} \leq \mathrm{E}\left[\int_{0}^{\infty}\left(\bar{f}\left(\boldsymbol{y}_{t}\right)-l\right)_{+} \mathrm{d} t\right]+\|\Psi\|_{\infty} .
$$


From Lemma 3.2, the right-hand side of (3.11) is bounded. In a similar way to the proof of Theorem 1.16 of [3], there exists a constant $\lambda_{1}$ such that

$$
\frac{1}{\varepsilon}\left\|\left(u_{\alpha}^{\varepsilon}-\Psi\right)_{-}\right\|_{L^{p, \gamma}} \leq\|f-l-(\alpha-\bar{L}) \Psi\|_{L^{p, \gamma}}+\lambda \lambda_{1}\left\|u_{\alpha}^{\varepsilon}-\Psi\right\|_{L^{p, \gamma}} .
$$

The right-hand side is bounded independently of $\varepsilon$ and $\alpha$ from the assumptions and (3.11). With the fact that $u_{\alpha}^{\varepsilon}$ satisfies

$$
(1-\bar{L}) u_{\alpha}^{\varepsilon}=(1-\alpha) u_{\alpha}^{\varepsilon}+\frac{1}{\varepsilon}\left(u_{\alpha}^{\varepsilon}-\Psi\right)_{-}+\bar{f}-l,
$$

we obtain a solution $\bar{u} \in W^{2, p, \gamma}$ of (3.6) as $\alpha, \varepsilon \rightarrow 0$. This completes the proof.

We prove the existence of an optimal stopping time for a conditional expectation rather than (3.1), which is needed to solve the main problem in this paper. Since the uniqueness of the solution has not been proved, we show the following lemma for the specific solution $\bar{u}$ obtained in the proof of Theorem 3.3, not for all the solutions of (3.6).

Lemma 3.7. Suppose that the assumptions of Theorem 3.3 hold. Let $\bar{u}$ be the solution of (3.6) obtained in Theorem 3.3. Let $\theta$ and $\theta^{\prime}$ be two stopping times with $\theta \leq \theta^{\prime}$ and $\mathrm{E}[\theta] \leq \mathrm{E}\left[\theta^{\prime}\right]<\infty$. Then

$$
\bar{u}\left(\boldsymbol{y}_{\theta}\right) \geq \mathrm{E}\left[\int_{\theta}^{\theta^{\prime}}\left(\bar{f}\left(\boldsymbol{y}_{s}\right)-l\right) \mathrm{d} s+\bar{u}\left(\boldsymbol{y}_{\theta^{\prime}}\right) \mid \mathcal{F}_{\theta}\right] .
$$

Set $\hat{\theta}:=\inf \left\{t>\theta \mid \Psi\left(\boldsymbol{y}_{t}\right) \geq \bar{u}\left(\boldsymbol{y}_{t}\right)\right\}$. Then $\mathrm{E}[\hat{\theta}]<\infty$ and

$$
\bar{u}\left(\boldsymbol{y}_{\theta}\right)=\mathrm{E}\left[\int_{\theta}^{\hat{\theta}}\left(\bar{f}\left(\boldsymbol{y}_{s}\right)-l\right) \mathrm{d} s+\bar{u}\left(\boldsymbol{y}_{\hat{\theta}}\right) \mid \mathcal{F}_{\theta}\right] .
$$

Proof. From (3.11), by passing $\alpha \rightarrow 0$ in (3.12), we obtain a solution $\bar{u}^{\varepsilon} \in W^{2, p, \gamma}$ of the following penalized equation:

$$
(1-\bar{L}) \bar{u}^{\varepsilon}-\frac{1}{\varepsilon}\left(\bar{u}^{\varepsilon}-\Psi\right)_{-}=\bar{u}^{\varepsilon}+\bar{f}-l,
$$

which is equivalent to

$$
-\bar{L} \bar{u}^{\varepsilon}-\frac{1}{\varepsilon}\left(\bar{u}^{\varepsilon}-\Psi\right)_{-}=\bar{f}-l .
$$

From Theorem 3.2 we have

$$
\bar{u}^{\varepsilon} \leq \bar{u}, \quad \bar{u}^{\varepsilon} \rightarrow \bar{u} \quad \text { in } C^{0}\left(\mathbb{R}^{d}\right) \text { as } \varepsilon \rightarrow 0 .
$$

Therefore, we can prove this lemma in a similar way to the proof of Lemma 3.6 of [4], as below.

Set $\mathcal{O}_{R}:=\left\{\boldsymbol{y} \in \mathbb{R}^{d}|| \boldsymbol{y} \mid<R\right\}$ and $\tau_{R}:=\inf \left\{t>0|| \boldsymbol{y}_{t} \mid \notin \mathcal{O}_{R}\right\}$. From the generalized Itô lemma (see [3, Theorem 8.5]) we obtain

$$
\begin{aligned}
\bar{u}^{\varepsilon}\left(\boldsymbol{y}_{\theta \wedge T \wedge \tau_{R}}\right)=\mathrm{E}_{\boldsymbol{y}}\left[\int_{\theta \wedge T \wedge \tau_{R}}^{\theta^{\prime} \wedge T \wedge \tau_{R}}\left(\bar{f}\left(\boldsymbol{y}_{S}\right)-l\right) \mathrm{d} s\right. \\
\quad+\frac{1}{\varepsilon} \int_{\theta \wedge T \wedge \tau_{R}}^{\theta^{\prime} \wedge T \wedge \tau_{R}}\left(\bar{u}^{\varepsilon}\left(\boldsymbol{y}_{S}\right)-\Psi\left(\boldsymbol{y}_{S}\right)\right)_{-} \mathrm{d} s+\bar{u}^{\varepsilon}\left(\boldsymbol{y}_{\theta^{\prime} \wedge T \wedge \tau_{R}}\right) \mid \mathcal{F}_{\left.\theta \wedge T \wedge \tau_{R}\right] .}
\end{aligned}
$$


From the assumptions we can use Lebesgue's convergence theorem. By passing $T, R \rightarrow \infty$ we obtain

$$
\begin{aligned}
\bar{u}^{\varepsilon}\left(\boldsymbol{y}_{\theta}\right) & =\mathrm{E}_{\boldsymbol{y}}\left[\int_{\theta}^{\theta^{\prime}}\left(\bar{f}\left(\boldsymbol{y}_{S}\right)-l\right) \mathrm{d} s+\frac{1}{\varepsilon} \int_{\theta}^{\theta^{\prime}}\left(\bar{u}^{\varepsilon}\left(\boldsymbol{y}_{s}\right)-\Psi\left(\boldsymbol{y}_{s}\right)\right)_{-} \mathrm{d} s+\bar{u}^{\varepsilon}\left(\boldsymbol{y}_{\theta^{\prime}}\right) \mid \mathcal{F}_{\theta}\right] \\
& \geq \mathrm{E}_{\boldsymbol{y}}\left[\int_{\theta}^{\theta^{\prime}}\left(\bar{f}\left(\boldsymbol{y}_{S}\right)-l\right) \mathrm{d} s+\bar{u}^{\varepsilon}\left(\boldsymbol{y}_{\theta^{\prime}}\right) \mid \mathcal{F}_{\theta}\right] .
\end{aligned}
$$

By sending $\varepsilon \rightarrow 0$ we obtain (3.13) from Lebesgue's convergence theorem.

Let $\hat{\theta}^{\varepsilon}:=\inf \left\{t \geq \theta \mid \Psi\left(\boldsymbol{y}_{t}\right) \geq \bar{u}^{\varepsilon}\left(\boldsymbol{y}_{t}\right)\right\}$. Note that $\hat{\theta}^{\varepsilon} \leq \hat{\theta}$. By setting $\theta=\theta \wedge T$ and $\theta^{\prime}=\hat{\theta}^{\varepsilon} \wedge T$ in (3.14), we obtain

$$
\begin{aligned}
\bar{u}^{\varepsilon}\left(\boldsymbol{y}_{\theta \wedge T}\right) & =\mathrm{E}_{\boldsymbol{y}}\left[\int_{\theta \wedge T}^{\hat{\theta}^{\varepsilon} \wedge T}\left(\bar{f}\left(\boldsymbol{y}_{s}\right)-l\right) \mathrm{d} s+\bar{u}^{\varepsilon}\left(\boldsymbol{y}_{\hat{\theta}^{\varepsilon} \wedge T}\right) \mid \mathcal{F}_{\theta}\right] \\
& \leq \mathrm{E}_{\boldsymbol{y}}\left[\int_{\theta \wedge T}^{\hat{\theta}^{\varepsilon} \wedge T}\left(\bar{f}\left(\boldsymbol{y}_{s}\right)-l\right) \mathrm{d} s+\bar{u}\left(\boldsymbol{y}_{\hat{\theta}^{\varepsilon} \wedge T}\right) \mid \mathcal{F}_{\theta}\right]
\end{aligned}
$$

from $\bar{u}^{\varepsilon} \leq \bar{u}$. In a similar way to the proof of Lemma 3.6 of [4], we can show that

$$
\hat{\theta}^{\varepsilon} \rightarrow \hat{\theta} \quad \text { a.s. }
$$

Again, by Lebesgue's convergence theorem we obtain

$$
\bar{u}\left(\boldsymbol{y}_{\theta \wedge T}\right) \leq \mathrm{E}_{\boldsymbol{y}}\left[\int_{\theta \wedge T}^{\hat{\theta} \wedge T}\left(\bar{f}\left(\boldsymbol{y}_{S}\right)-l\right) \mathrm{d} s+\bar{u}\left(\boldsymbol{y}_{\theta \hat{\wedge} T}\right) \mid \mathcal{F}_{\theta}\right] .
$$

From Lemma 3.4, it follows that $\mathrm{E}[\hat{\theta}]<\infty$. By sending $T \rightarrow \infty$ we obtain the conclusion.

From the above lemma we obtain an optimal stopping time for (3.1) by setting $\theta \equiv 0$. Let $\tau^{*}:=\inf \left\{t>0 \mid \Psi\left(\boldsymbol{y}_{t}\right) \geq \bar{u}\left(\boldsymbol{y}_{t}\right)\right\}$.

Theorem 3.4. Suppose that the assumptions of Theorem 3.3 hold. Then we have

$$
\begin{aligned}
\bar{u}(\boldsymbol{y}) & =\sup _{\tau} \mathrm{E}_{\boldsymbol{y}}\left[\int_{0}^{\tau}\left(\bar{f}\left(\boldsymbol{y}_{S}\right)-l\right) \mathrm{d} s+\Psi\left(\boldsymbol{y}_{\tau}\right)\right] \\
& =\mathrm{E}_{\boldsymbol{y}}\left[\int_{0}^{\tau^{*}}\left(\bar{f}\left(\boldsymbol{y}_{s}\right)-l\right) \mathrm{d} s+\Psi\left(\boldsymbol{y}_{\tau^{*}}\right)\right] .
\end{aligned}
$$

\section{QVI of 'ergodic' type}

In this section we shall show the existence of a solution for the following QVI of 'ergodic' type:

$$
\varepsilon_{0}(v, w-v) \geq(\bar{f}-l, w-v), \quad v(\boldsymbol{y}) \geq \mathcal{M} v(\boldsymbol{y})
$$

for all $w \geq \mathcal{M} v$ and $v, w \in W^{1,2, \gamma}\left(\mathbb{R}^{d}\right)$, where we define $\mathcal{M} v(\boldsymbol{y})$ as

$$
\mathcal{M} v(\boldsymbol{y})=\sup _{\zeta \in \mathbb{R}^{d}} v(\boldsymbol{y}+\zeta)+\bar{C}(\boldsymbol{y}, \zeta)
$$


If $v$ is bounded from above, $\mathcal{M} v$ makes sense. We can see that if $u \leq v$ then $\mathcal{M} u \leq \mathcal{M} v$. We can see from (2.10) that

$$
0>\bar{C}(\boldsymbol{y}, \boldsymbol{z}-\boldsymbol{y}) \geq \bar{C}(\boldsymbol{y}, \boldsymbol{x}-\boldsymbol{y})+\bar{C}(\boldsymbol{x}, \boldsymbol{z}-\boldsymbol{x})+\left|\log \left(1-c_{0}\right)\right|
$$

for any $\boldsymbol{x}, \boldsymbol{y}, \boldsymbol{z} \in \mathbb{R}^{d}$. By virtue of $C(\boldsymbol{h}, \boldsymbol{\xi}), \mathcal{M} v$ is Lipschitz continuous. For this type of equation, the pair of a function $v$ and a constant $l$ is considered as a solution.

Definition 4.1. Let $v \in W^{1,2, \gamma}\left(\mathbb{R}^{d}\right)$, and let $l \in \mathbb{R}$. We say that a pair $(v, l)$ is a solution of the QVI (4.1) if the pair $(v, l)$ satisfies (4.1).

We cannot directly solve the QVI (4.1). We use perturbation methods. Let $u_{\alpha}$ be the solution of a QVI with a discount factor $\alpha$ in the following theorem. In perturbation methods we take the limit of $\left(u_{\alpha}-\inf _{\boldsymbol{y} \in \mathbb{R}^{d}} u_{\alpha}(\boldsymbol{y}), \alpha \inf _{\boldsymbol{y} \in \mathbb{R}^{d}} u_{\alpha}(\boldsymbol{y})\right)$ as $\alpha \rightarrow 0$ and prove that the pair converges to a certain $(v, l)$, which is the solution of the QVI of 'ergodic' type, (4.1).

Theorem 4.1. ([4, p. 415].) Suppose that $\alpha>0$. Then there exists a unique solution $u_{\alpha} \in$ $W^{1,2, \gamma}\left(\mathbb{R}^{d}\right)$ of the following $Q V I$ :

$$
\mathcal{E}_{\alpha}\left(u_{\alpha}, v-u_{\alpha}\right) \geq\left(\bar{f}, v-u_{\alpha}\right), \quad u_{\alpha}(\boldsymbol{y}) \geq \mathcal{M} u_{\alpha}(\boldsymbol{y})
$$

for all $v \geq \mathcal{M} u_{\alpha}$ and $v, w \in W^{1,2, \gamma}\left(\mathbb{R}^{d}\right)$. This solution is continuous and bounded. We also have

$$
u_{\alpha}(\boldsymbol{y})=\sup _{\tau} \mathrm{E}_{\boldsymbol{y}}\left[\int_{0}^{\tau} \mathrm{e}^{-\alpha s} \bar{f}\left(\boldsymbol{y}_{s}\right) \mathrm{d} s+\mathrm{e}^{-\alpha \tau} \mathcal{M} u\left(\boldsymbol{y}_{\tau}\right)\right],
$$

where $\boldsymbol{y}_{t}$ is the solution of (2.15).

Proof. We obtain the existence and the continuity of the solution from Theorem 4.1 of [4]. Since $\bar{f}$ is bounded and continuous, we can use Hanouzet-Joly's $L^{\infty}$ technique, described in Lemma 1.4 of [4], to prove the uniqueness of the solution, even though the domain is unbounded. So, we obtain the existence and the uniqueness of the solution and its continuity. Here $\mathcal{M} u_{\alpha}$ is Lipschitz continuous by virtue of $\mathcal{M}$. Hence, we obtain (4.3) from Theorem 3.1.

Let $v_{\alpha}(\boldsymbol{y}):=u_{\alpha}(\boldsymbol{y})-\inf _{\boldsymbol{x} \in \mathbb{R}^{d}} u_{\alpha}(\boldsymbol{x})$. Let $l_{\alpha}$ denote $\alpha \inf _{\boldsymbol{x} \in \mathbb{R}^{d}} u_{\alpha}(\boldsymbol{x})$. Then $v_{\alpha}$ satisfies the following QVI:

$$
\mathcal{E}_{\alpha}\left(v_{\alpha}, w-v_{\alpha}\right) \geq\left(\bar{f}-l_{\alpha}, w-v_{\alpha}\right), \quad v_{\alpha}(\boldsymbol{y}) \geq \mathcal{M} v_{\alpha}(\boldsymbol{y})
$$

for all $w \geq \mathcal{M} v_{\alpha}$ and $v, w \in W^{1,2, \gamma}\left(\mathbb{R}^{d}\right)$. Therefore, by Theorem 4.1, we have the following probabilistic representation of $v_{\alpha}$ :

$$
v_{\alpha}(\boldsymbol{y})=\sup _{\tau} \mathrm{E}_{\boldsymbol{y}}\left[\int_{0}^{\tau} \mathrm{e}^{-\alpha s}\left(\bar{f}\left(\boldsymbol{y}_{s}\right)-l_{\alpha}\right) \mathrm{d} s+\mathrm{e}^{-\alpha \tau} \mathcal{M} v_{\alpha}\left(\boldsymbol{y}_{\tau}\right)\right] .
$$

In this section, taking a subsequence if needed, we shall show that $\left(v_{\alpha}, l_{\alpha}\right)$ converges to a certain pair $(v, \bar{l})$ as $\alpha \rightarrow 0$.

We obtain the upper and lower bounds of $\lim \inf l_{\alpha}$, which implies the convergence of a subsequence of $l_{\alpha}$. Let $l^{*}$ be the constant defined in (2.13).

Lemma 4.1. We have

$$
\sup _{\boldsymbol{y} \in \mathbb{R}^{d}} \bar{f}(\boldsymbol{y}) \geq \limsup _{\alpha \rightarrow 0} l_{\alpha} \geq \liminf _{\alpha \rightarrow 0} l_{\alpha} \geq l^{*} \geq \bar{\beta} .
$$


Proof. Suppose that $l_{\alpha}>\sup \bar{f}(\boldsymbol{y})$ for a certain $\alpha$. Fix $0<\varepsilon<-\log \left(1-c_{0}\right)$. There exists $\boldsymbol{y}_{0} \in \mathbb{R}^{d}$ such that $v_{\alpha}\left(\boldsymbol{y}_{0}\right)+\varepsilon>\sup v_{\alpha}(\boldsymbol{y})$, because $v_{\alpha}$ is bounded and continuous. By the definition of $\mathcal{M}$ we obtain

$$
v_{\alpha}\left(\boldsymbol{y}_{0}\right)+\varepsilon+\log \left(1-c_{0}\right)>\sup _{\boldsymbol{y} \in \mathbb{R}^{d}} \mathcal{M} v_{\alpha}(\boldsymbol{y}) .
$$

From (4.5) and $\bar{f}(\boldsymbol{y})-l_{\alpha}<0$, we obtain

$$
v_{\alpha}\left(\boldsymbol{y}_{0}\right) \leq \sup _{\boldsymbol{y} \in \mathbb{R}^{d}} \mathcal{M} v_{\alpha}(\boldsymbol{y})<v_{\alpha}\left(\boldsymbol{y}_{0}\right)+\varepsilon+\log \left(1-c_{0}\right)
$$

and

$$
0<-\log \left(1-c_{0}\right)<\varepsilon
$$

This is a contradiction. Therefore, we obtain $\sup \bar{f}(\boldsymbol{y}) \geq \lim \sup l_{\alpha}$.

The second inequality is trivial.

Fix any $\delta>0$. By the definition of $l^{*}$, there exists a strategy $\left\{\left(\tau_{n}, \zeta_{n}\right)\right\}_{n=1}^{\infty}$ such that

$$
l^{*}-\frac{1}{8} \delta \leq \liminf _{T \rightarrow \infty} \frac{1}{T} \mathrm{E}\left[\int_{0}^{T} \bar{f}\left(\boldsymbol{y}_{s}\right) \mathrm{d} s+\sum_{n=1}^{\infty} \bar{C}\left(\boldsymbol{y}_{\tau_{n}-}, \zeta_{n}\right) 1_{\left\{\tau_{n}<T\right\}}\right] .
$$

We can take large enough $T$ such that $\|\bar{C}(\cdot, \cdot)\|_{\infty}<\delta T / 4$ and

$$
l^{*}-\frac{1}{4} \delta \leq \frac{1}{T} \mathrm{E}\left[\int_{0}^{T} \bar{f}\left(\boldsymbol{y}_{S}\right) \mathrm{d} s+\sum_{n=1}^{\infty} \bar{C}\left(\boldsymbol{y}_{\tau_{n}-}, \zeta_{n}\right) 1_{\left\{\tau_{n}<T\right\}}\right] .
$$

It follows that

$$
\left(l^{*}-\frac{1}{2} \delta\right) T \leq \mathrm{E}_{\boldsymbol{y}}\left[\int_{0}^{T} \bar{f}\left(\boldsymbol{y}_{s}\right) \mathrm{d} s+\sum_{n=1}^{\infty} \bar{C}\left(\boldsymbol{y}_{\tau_{n}-}, \zeta_{n}\right) 1_{\left\{\tau_{n}<T\right\}}+\bar{C}\left(\boldsymbol{y}_{T}, \boldsymbol{y}-\boldsymbol{y}_{T}\right)\right] .
$$

Hence, there exists $\alpha_{0}>0$ such that, for any positive $\alpha<\alpha_{0}$,

$$
\begin{aligned}
\int_{0}^{T} \mathrm{e}^{-\alpha s}\left(l^{*}-\delta\right) \mathrm{d} s \leq \mathrm{E}_{\boldsymbol{y}}\left[\int_{0}^{T} \mathrm{e}^{-\alpha s} \bar{f}\left(\boldsymbol{y}_{S}\right) \mathrm{d} s+\sum_{n=1}^{\infty} \exp \left\{-\alpha \tau_{n}\right\} \bar{C}\left(\boldsymbol{y}_{\tau_{n}-}, \zeta_{n}\right) 1_{\left\{\tau_{n}<T\right\}}\right. \\
\left.+\mathrm{e}^{-\alpha T} \bar{C}\left(\boldsymbol{y}_{T}, \boldsymbol{y}-\boldsymbol{y}_{T}\right)\right] .
\end{aligned}
$$

With the standard argument of impulsive control theory and the above inequality, we obtain

$$
\begin{aligned}
u_{\alpha}(\boldsymbol{y}) \geq \mathrm{E}_{\boldsymbol{y}}\left[\int_{0}^{T} \mathrm{e}^{-\alpha s} \bar{f}\left(\boldsymbol{y}_{s}\right) \mathrm{d} s+\sum_{n=1}^{\infty} \exp \left\{-\alpha \tau_{n}\right\} \bar{C}\left(\boldsymbol{y}_{\tau_{n}-}, \zeta_{n}\right) 1_{\left\{\tau_{n}<T\right\}}+\mathrm{e}^{-\alpha T} u_{\alpha}\left(\boldsymbol{y}_{T}\right)\right] \\
\geq \mathrm{E}_{\boldsymbol{y}}\left[\int_{0}^{T} \mathrm{e}^{-\alpha s} \bar{f}\left(\boldsymbol{y}_{s}\right) \mathrm{d} s+\sum_{n=1}^{\infty} \exp \left\{-\alpha \tau_{n}\right\} \bar{C}\left(\boldsymbol{y}_{\tau_{n}-}, \zeta_{n}\right) 1_{\left\{\tau_{n}<T\right\}}\right. \\
\left.\quad+\mathrm{e}^{-\alpha T}\left(\overline{\boldsymbol{C}}\left(\boldsymbol{y}_{T}, \boldsymbol{y}-\boldsymbol{y}_{T}\right)+u_{\alpha}(\boldsymbol{y})\right)\right] \\
\geq \int_{0}^{T} \mathrm{e}^{-\alpha s}\left(l^{*}-\delta\right) \mathrm{d} s+\mathrm{e}^{-\alpha T} u_{\alpha}(\boldsymbol{y}) .
\end{aligned}
$$


Therefore, we obtain

$$
\alpha u_{\alpha}(\boldsymbol{y}) \geq l^{*}-\delta
$$

This implies the third inequality.

Now we prove the last inequality. Let $v_{t}$ denote the solution of the SDE (2.9). It follows that

$$
\mathrm{E}\left[\log v_{T}\right] \geq \mathrm{E}\left[\log v_{0}+\log \left(\exp \left\{\left(b^{i}-\frac{\left(\sigma \sigma^{\top}\right)^{i i}}{2}\right) T+\left(\sigma W_{T}\right)^{i}\right\}\right)\right]=\left(\beta^{i}+r\right) T+\log v_{0}
$$

and

$$
\mathrm{E}\left[\log v_{T}\right] \geq \mathrm{E}\left[\log v_{0}+\log \mathrm{e}^{r T}\right]=r T+\log v_{0} .
$$

From the definition of $l^{*}$,

$$
l^{*} \geq \liminf _{T \rightarrow \infty} \frac{1}{T} \mathrm{E}\left[\log v_{T}\right]-r \geq \bar{\beta} .
$$

We obtain the result.

From the above lemma we can obtain an optimal strategy for a certain case.

Proposition 4.1. Assume that $\lim _{\inf _{\alpha \rightarrow 0}} l_{\alpha}=\bar{\beta}$. Then the optimal growth rate $\rho$ satisfies

$$
\rho=\bar{\beta}+r=r \vee \max _{1 \leq i \leq d}\left(b^{i}-\frac{1}{2}\left(\sigma \sigma^{\top}\right)^{i i}\right),
$$

and the optimal strategy is to buy and hold those assets that attain $\bar{\beta}+r$. In other words, buying and holding the ith risky asset is optimal if $\bar{\beta}=\left(b^{i}-r-\left(\sigma \sigma^{\top}\right)^{i i} / 2\right)$.

In some special cases the assumption of the above proposition holds. If $f(\boldsymbol{h})$ attains the maximum at some $\boldsymbol{e}_{i}$ then $\bar{\beta}=\beta_{i}$ and $\sup \bar{f}(\cdot)=\bar{l}=\bar{\beta}$ hold.

\subsection{Perturbation method and the existence of the solution}

It holds from Lemma 4.1 that $\bar{l}:=\lim \sup l_{\alpha}<\infty$. When $\bar{l}=\bar{\beta}$, we can obtain an optimal strategy, as in Proposition 4.1. So, we suppose that $\bar{l}>\bar{\beta}$ in this subsection and in the next section.

From the definition of $v_{\alpha}$ we have $\left\|v_{\alpha}\right\|_{\infty} \leq\|C(\cdot, \cdot)\|_{\infty}$. Since $\mathcal{M} v_{\alpha}$ is Lipschitz continuous, independently of $\alpha$, we can obtain the boundedness of $v_{\alpha}$ in $W^{1, \infty, \gamma}\left(\mathbb{R}^{d}\right)$ in the same way as in Theorem 3.3. Therefore, there exists a subsequence $v_{\alpha_{k}}$ that converges to a certain bounded function $\bar{v}$ weakly in $W^{1, p, \gamma}\left(\mathbb{R}^{d}\right)$ and strongly in $L^{p, \gamma}\left(\mathbb{R}^{d}\right)$ as $\alpha_{k} \rightarrow 0$.

Suppose that $v_{\alpha_{k}} \rightarrow \bar{v}$ in $L^{\infty}\left(\mathbb{R}^{d}\right)$. Then we have $\mathcal{M} v_{\alpha_{k}} \rightarrow \mathcal{M} \bar{v}$ in $L^{\infty}\left(\mathbb{R}^{d}\right)$. Fix $\delta>0$. There exists $\alpha_{0}$ such that, for all $\alpha_{k}<\alpha_{0}$,

$$
w \geq \mathcal{M} \bar{v} \Longrightarrow w+\delta \geq \mathcal{M} v_{\alpha_{k}} .
$$

Let $w^{\delta}:=w+\delta$. By setting $w=w^{\delta}$ in (4.4) and sending $\alpha_{k} \rightarrow 0$, we obtain

$$
\varepsilon_{0}\left(\bar{v}, w^{\delta}-\bar{v}\right) \geq\left(\bar{f}-\bar{l}, w^{\delta}-\bar{v}\right), \quad \bar{v}(\boldsymbol{y}) \geq \mathcal{M} \bar{v}(\boldsymbol{y})
$$

for all $w \geq \mathcal{M} \bar{v}$ and $w \in W^{1,2, \gamma}\left(\mathbb{R}^{d}\right)$, in the same way as in Theorem 3.3. By taking the limit $\delta \rightarrow 0$ we obtain

$$
\varepsilon_{0}(\bar{v}, w-\bar{v}) \geq(\bar{f}-\bar{l}, w-\bar{v}), \quad \bar{v}(\boldsymbol{y}) \geq \mathcal{M} \bar{v}(\boldsymbol{y}),
$$

for all $w \geq \mathcal{M} \bar{v}$ and $w \in W^{1,2, \gamma}\left(\mathbb{R}^{d}\right)$. 
In what follows we shall prove that $v_{\alpha_{k}} \rightarrow \bar{v}$ in $L^{\infty}\left(\mathbb{R}^{d}\right)$ and obtain the solution above by taking a subsequence if needed. Recall that $\phi(y):=\psi^{-1}(\boldsymbol{y})$ defined in (2.14). An elementary calculation shows the following lemma.

Lemma 4.2. Fix any $\varepsilon>0$ and $R>0$. Then there exists a constant $\delta$ such that

$$
|\phi(\boldsymbol{x})-\phi(\boldsymbol{y})|<\delta \Longrightarrow|\phi(\boldsymbol{x}+\boldsymbol{v})-\phi(\boldsymbol{y}+\boldsymbol{v})|<\varepsilon
$$

for any $\boldsymbol{v}, \boldsymbol{x}, \boldsymbol{y} \in \mathbb{R}^{d}$ and $|\boldsymbol{v}|<R$.

Theorem 4.2. Suppose that (3.2) holds and that $\bar{l}>\bar{\beta}$. Then we have

$$
v_{\alpha_{k}} \rightarrow \bar{v} \text { in } L^{\infty}\left(\mathbb{R}^{d}\right),
$$

by taking a subsequence if needed, and $(\bar{v}, \bar{l})$ is a solution of (4.1).

Proof. It is sufficient to prove that

$$
v_{\alpha_{k}} \circ \psi \rightarrow \tilde{v} \text { in } L^{\infty}(\Delta)
$$

for some $\tilde{v}$. In fact, if (4.6) holds, we have

$$
\begin{aligned}
\left\|v_{\alpha_{k}}-\tilde{v} \circ \psi^{-1}\right\|_{\infty} & =\left\|v_{\alpha_{k}} \circ \psi \circ \psi^{-1}-\tilde{v} \circ \psi^{-1}\right\|_{\infty} \\
& =\left\|v_{\alpha_{k}} \circ \psi-\tilde{v}\right\|_{\infty} \\
& \rightarrow 0
\end{aligned}
$$

and $\tilde{v} \circ \psi^{-1}=\bar{v}$ must hold. We prove (4.6) by using Ascoli-Arzela's theorem.

Let $\tilde{v}_{\alpha}(\boldsymbol{h})=v_{\alpha} \circ \psi(\boldsymbol{h})$ and $\sigma_{R}(\boldsymbol{y})=\left\{t>0|| \boldsymbol{y}_{t}-\boldsymbol{y}_{0} \mid \geq R, \boldsymbol{y}_{0}=\boldsymbol{y}\right\}$. Fix any $\varepsilon>0$. There exists a constant $R$ such that $\mathrm{P}\left(\sigma_{R}(\boldsymbol{y})<\tau_{\alpha}^{*}(\boldsymbol{y})\right)<\varepsilon$ for any $\boldsymbol{y} \in \mathbb{R}^{d}$. Let $\boldsymbol{y}_{t}$ and $\boldsymbol{y}_{t}^{\prime}$ be solutions of the SDE (2.15) with initial values $\boldsymbol{y}$ and $\boldsymbol{y}^{\prime}$, respectively. Note that $\left|\boldsymbol{y}_{t}-\boldsymbol{y}\right|=\left|\boldsymbol{y}_{t}^{\prime}-\boldsymbol{y}^{\prime}\right| \leq R$ for all $t \leq \sigma_{R}(\boldsymbol{y})$. From Lemma 4.2 and the uniform continuity of $f$, there exists a constant $\delta_{0}$ such that if $\left|\phi(\boldsymbol{y})-\phi\left(\boldsymbol{y}^{\prime}\right)\right|<\delta_{0}$ then

$$
\left|\bar{f}\left(\boldsymbol{y}_{s}\right)-\bar{f}\left(\boldsymbol{y}_{s}^{\prime}\right)\right|=\left|f \circ \phi\left(\boldsymbol{y}_{s}\right)-f \circ \phi\left(\boldsymbol{y}_{s}^{\prime}\right)\right|<\varepsilon
$$

on $t \leq \sigma_{R}(\boldsymbol{y})$. By virtue of $\mathcal{M}$, there exists a constant $\delta_{1}$ such that if $\left|\phi(\boldsymbol{y})-\phi\left(\boldsymbol{y}^{\prime}\right)\right|<\delta_{1}$ then

$$
\left|\mathcal{M} v_{\alpha}\left(\boldsymbol{y}_{t}\right)-\mathcal{M} v_{\alpha}\left(\boldsymbol{y}_{t}^{\prime}\right)\right| \leq \sup _{\boldsymbol{x} \in \mathbb{R}^{d}}\left|c\left(\phi\left(\boldsymbol{y}_{t}\right), \phi(\boldsymbol{x})-\phi\left(\boldsymbol{y}_{t}\right)\right)-c\left(\phi\left(\boldsymbol{y}_{t}^{\prime}\right), \phi(\boldsymbol{x})-\phi\left(\boldsymbol{y}_{t}^{\prime}\right)\right)\right|<\varepsilon
$$

on $t \leq \sigma_{R}(\boldsymbol{y})$. Set $\delta=\delta_{0} \wedge \delta_{1}$. Note that $\left\|\mathcal{M} v_{\alpha}(\boldsymbol{y})-\mathcal{M} v_{\alpha}\left(\boldsymbol{y}^{\prime}\right)\right\|_{\infty} \leq\|C(\cdot, \cdot)\|_{\infty}$. Let $\boldsymbol{h}$ and $\boldsymbol{h}^{\prime}$ denote $\phi(\boldsymbol{y})$ and $\phi\left(\boldsymbol{y}^{\prime}\right)$, respectively. We find that if $\left|\boldsymbol{h}-\boldsymbol{h}^{\prime}\right|<\delta$ then

$$
\begin{aligned}
v_{\alpha} \circ \psi(\boldsymbol{h})-v_{\alpha} \circ \psi\left(\boldsymbol{h}^{\prime}\right)= & v_{\alpha}(\boldsymbol{y})-v_{\alpha}\left(\boldsymbol{y}^{\prime}\right) \\
\leq & \mathrm{E}\left[\int_{0}^{\sigma_{R} \wedge \tau_{\alpha}^{*}(\boldsymbol{y})}\left(\bar{f}\left(\boldsymbol{y}_{s}\right)-\bar{f}\left(\boldsymbol{y}_{s}^{\prime}\right) \mathrm{d} s\right) \mathrm{e}^{-\alpha s} \mathrm{~d} s\right. \\
& \left.\quad+\exp \left\{-\alpha\left(\sigma_{R} \wedge \tau_{\alpha}^{*}(\boldsymbol{y})\right)\right\}\left(\mathcal{M} v_{\alpha}\left(\boldsymbol{y}_{\sigma_{R} \wedge \tau_{\alpha}^{*}(\boldsymbol{y})}\right)-\mathcal{M} v_{\alpha}\left(\boldsymbol{y}_{\sigma_{R} \wedge \tau_{\alpha}^{*}(\boldsymbol{y})}^{\prime}\right)\right)\right] \\
\leq & \frac{\varepsilon}{\alpha} \mathrm{E}\left[1-\exp \left\{-\alpha\left(\sigma_{R} \wedge \tau_{\alpha}^{*}(\boldsymbol{y})\right)\right\}\right] \\
& +\mathrm{E}\left[\exp \left\{-\alpha \sigma_{R}\right\}\left(\mathcal{M} v_{\alpha}\left(\boldsymbol{y}_{\sigma_{R}}\right)-\mathcal{M} v_{\alpha}\left(\boldsymbol{y}_{\sigma_{R}}^{\prime}\right)\right) ; \sigma_{R}<\tau_{\alpha}^{*}(\boldsymbol{y})\right] \\
& +\mathrm{E}\left[\exp \left\{-\alpha \tau_{\alpha}^{*}(\boldsymbol{y})\right\}\left(\mathcal{M} v_{\alpha}\left(\boldsymbol{y}_{\tau_{\alpha}^{*}(\boldsymbol{y})}\right)-\mathcal{M} v_{\alpha}\left(\boldsymbol{y}_{\tau_{\alpha}^{*}(\boldsymbol{y})}^{\prime}\right)\right) ; \sigma_{R} \geq \tau_{\alpha}^{*}(\boldsymbol{y})\right] \\
\leq & \varepsilon K+\varepsilon\|C(\cdot, \cdot)\|_{\infty}+\varepsilon .
\end{aligned}
$$


We can obtain the same inequality when $\boldsymbol{h}$ and $\boldsymbol{h}^{\prime}$ are interchanged. Hence, we can naturally define $v_{\alpha} \circ \psi(\cdot)$ on $\partial \Delta$. It follows that $v_{\alpha} \circ \psi(\cdot)$ is equicontinuous and bounded on $\Delta$. By Ascoli-Arzela's theorem we obtain the desired conclusion.

We can see that $\bar{v}$ is a solution of the VI (3.6) when $\Psi=\mathcal{M} \bar{v}$ is set. But recall that we have not been able to prove the uniqueness of the solution of (3.6). We have only proved that a solution of (3.6) exists and can be used to derive an optimal stopping time for (3.1). When we apply Lemma 3.7 to $\bar{v}$, we need to show that $\bar{v}$ in Theorem 4.2 coincides with $u$ obtained in the proof of Theorem 3.3.

Let $u_{\alpha}$ be the solution of the VI (3.7) with $\Psi=\mathcal{M} \bar{v}$ and $l=\bar{l}$. Then $u_{\alpha}$ has the following stochastic representation:

$$
u_{\alpha}(\boldsymbol{y})=\sup _{\tau} \mathrm{E}\left[\int_{0}^{\tau} \mathrm{e}^{-\alpha s}\left(\bar{f}\left(\boldsymbol{y}_{s}\right)-\bar{l}\right) \mathrm{d} s+\mathrm{e}^{-\alpha \tau} \mathcal{M} \bar{v}\left(\boldsymbol{y}_{\tau}\right)\right] .
$$

We obtain

$$
\begin{aligned}
v_{\alpha}(\boldsymbol{y})-u_{\alpha}(\boldsymbol{y}) & \leq \mathrm{E}\left[\int_{0}^{\tau^{*}(\boldsymbol{y})} \mathrm{e}^{-\alpha s}\left(l_{\alpha}-\bar{l}\right) \mathrm{d} s+\mathrm{e}^{-\alpha \tau^{*}(\boldsymbol{y})}\left(\mathcal{M} v_{\alpha}\left(\boldsymbol{y}_{\tau^{*}}\right)-\mathcal{M} \bar{v}\left(\boldsymbol{y}_{\tau^{*}}\right)\right)\right] \\
& \leq\left|l_{\alpha}-\bar{l}\right| K+\left\|\mathcal{M} v_{\alpha}-\mathcal{M} \bar{v}\right\|_{\infty} .
\end{aligned}
$$

When $v_{\alpha}$ and $u_{\alpha}$ are interchanged, we can obtain the same inequality. Therefore,

$$
\left\|v_{\alpha_{k}}-u_{\alpha_{k}}\right\|_{\infty} \rightarrow 0 \quad \text { as } \alpha_{k} \rightarrow 0 .
$$

Hence, we obtain the desired result.

\section{Deriving an optimal strategy}

In this section we derive an optimal strategy by using Lemma 3.7 and the solution of the QVI obtained in Theorem 4.2.

Lemma 5.1. Fix any admissible proportional strategy $\left\{\left(\tau_{n}, \boldsymbol{\xi}_{n}\right)\right\}_{n=1}^{\infty} \in \mathcal{A}$. Suppose that $\mathrm{P}\left(\lim _{n \rightarrow \infty} \tau_{n}<\infty\right)>0$. Then there exists a constant $T_{0}$ such that, for all $T>T_{0}$,

$$
\mathrm{E}_{\boldsymbol{h}}\left[\int_{0}^{T}\left(f\left(\boldsymbol{h}_{s}\right)+r\right) \mathrm{d} s+\sum_{n=1}^{\infty} C\left(\boldsymbol{h}_{\tau_{n}-}, \boldsymbol{\xi}_{n}\right) 1_{\left\{\tau_{n}<T\right\}}\right]=-\infty
$$

Proof. From the assumption, there exist $\delta$ and $T_{0}$ such that, for all $T>T_{0}, \mathrm{P}\left(\lim _{n \rightarrow \infty} \tau_{n}<\right.$ $T)>\delta$. But it holds that, on $\left\{\omega \in \Omega \mid \lim _{n \rightarrow \infty} \tau_{n}<T\right\}$,

$$
\begin{aligned}
\int_{0}^{T}\left(f\left(\boldsymbol{h}_{s}\right)+r\right) \mathrm{d} s+\sum_{n=1}^{\infty} C\left(\boldsymbol{h}_{\tau_{n}-}, \boldsymbol{\xi}_{n}\right) 1_{\left\{\tau_{n}<T\right\}} & \leq T\|f+r\|_{\infty}+\sum_{n=1}^{\infty} \log \left(1-c_{0}\right) \\
& =-\infty
\end{aligned}
$$

We obtain the result.

Hence, it is sufficient to consider only admissible strategies that satisfy $\lim _{n \rightarrow \infty} \tau_{n}=\infty$ a.s. 
Theorem 5.1. Assume that the same assumptions as in Theorem 4.2 hold. Let $\bar{v}$ be the solution of the QVI (4.1) obtained in Theorem 4.2. We define a strategy $\hat{a}_{d m}:=\left\{\left(\hat{\tau}_{n}, \hat{\zeta}_{n}\right)\right\}_{n=1}^{\infty}$ as follows:

$$
\hat{\tau}_{1}=\inf \left\{t>0 \mid \mathcal{M} \bar{v}\left(\boldsymbol{y}_{t}\right) \geq \bar{v}\left(\boldsymbol{y}_{t}\right)\right\}, \quad \hat{\tau}_{n}=\inf \left\{t>\hat{\tau}_{n-1} \mid \mathcal{M} \bar{v}\left(\boldsymbol{y}_{t}\right) \geq \bar{v}\left(\boldsymbol{y}_{t}\right)\right\} .
$$

Fix $\delta, 0<\delta<\left(\left|\log \left(1-c_{0}\right)\right| \wedge 1\right) / 2$. Take $\hat{\zeta}_{n}$ that attains

$$
\bar{v}\left(\boldsymbol{y}_{\hat{\tau}_{n}-}+\hat{\zeta}_{n}\right)+\bar{C}\left(\boldsymbol{y}_{\hat{\tau}_{n}-}, \hat{\zeta}_{n}\right)+\frac{\delta}{2^{n}} \geq \mathcal{M} \bar{v}\left(\boldsymbol{y}_{\hat{\tau}_{n}-}\right)=\bar{v}\left(\boldsymbol{y}_{\hat{\tau}_{n}-}\right) .
$$

Then $\hat{a}_{d m}$ is an optimal admissible strategy for the portfolio optimization problem, (2.3), which attains the optimal growth rate $\rho$. It holds that

$$
\rho=r+\bar{l} \text {. }
$$

Proof. Fix any admissible strategy $\tilde{a}_{d m}=\left\{\left(\tau_{n}, \zeta_{n}\right)\right\}_{n=1}^{\infty} \in \tilde{\mathcal{A}}$. From Lemma 3.7 we obtain

$$
\begin{aligned}
\bar{v}\left(\boldsymbol{y}_{\tau_{n} \wedge T}\right) & \geq \mathrm{E}\left[\int_{\tau_{n} \wedge T}^{\tau_{n+1} \wedge T}\left(\bar{f}\left(\boldsymbol{y}_{S}\right)-\bar{l}\right) \mathrm{d} s+\bar{v}\left(\boldsymbol{y}_{T \wedge \tau_{n+1}-}\right) \mid \mathcal{F}_{\tau_{n}}\right] \\
& \geq \mathrm{E}\left[\int_{\tau_{n} \wedge T}^{\tau_{n+1} \wedge T}\left(\bar{f}\left(\boldsymbol{y}_{S}\right)-\bar{l}\right) \mathrm{d} s+\bar{v}\left(\boldsymbol{y}_{\tau_{n+1} \wedge T}\right)+\bar{C}\left(\boldsymbol{y}_{T \wedge \tau_{n+1}-}, \zeta_{n+1}\right) \mid \mathcal{F}_{\tau_{n}}\right] .
\end{aligned}
$$

By summing up both sides and sending $n \rightarrow \infty$, we obtain

$$
\bar{v}(\boldsymbol{y})-E_{\boldsymbol{h}}\left[\bar{v}\left(\boldsymbol{y}_{T}\right)\right] \geq \mathrm{E}\left[\int_{0}^{T}\left(\bar{f}\left(\boldsymbol{y}_{s}\right)-\bar{l}\right) \mathrm{d} s+\sum_{n=1}^{\infty} \bar{C}\left(\boldsymbol{y}_{T \wedge \tau_{n+1}-}, \zeta_{n+1}\right) 1_{\left\{\tau_{n}<T\right\}}\right] .
$$

Hence, we obtain the following upper bound of the optimal growth rate $\rho$ :

$$
\begin{aligned}
J\left(\tilde{a}_{d m}\right) & =r+\liminf _{T \rightarrow \infty} \frac{1}{T} \mathrm{E}\left[\int_{0}^{T} \bar{f}\left(\boldsymbol{y}_{s}\right) \mathrm{d} s+\sum_{n=1}^{\infty} \bar{C}\left(\boldsymbol{y}_{T \wedge \tau_{n+1}-}, \zeta_{n+1}\right) 1_{\left\{\tau_{n}<T\right\}}\right] \\
& \leq r+\bar{l}+\liminf _{T \rightarrow \infty} \frac{2\|\bar{v}\|_{\infty}}{T} \\
& =r+\bar{l} .
\end{aligned}
$$

Here we show that the strategy $\hat{a}_{d m}$ is admissible. It is sufficient to prove that $\hat{\tau}_{n} \rightarrow \infty$ a.s. and $\hat{\tau}_{n}<\hat{\tau}_{n+1}$ a.s. The former can be obtained in the same way as in Lemma 5.1. We show the latter. Since $\bar{v} \geq \mathcal{M} \bar{v}$, it follows that

$$
\bar{v}\left(\boldsymbol{y}_{\hat{\tau}_{n}-}\right) \geq \bar{v}\left(\boldsymbol{y}_{\hat{\tau}_{n}-}+\hat{\zeta}_{n}+\zeta\right)+\bar{C}\left(\boldsymbol{y}_{\hat{\tau}_{n}-}, \hat{\zeta}_{n}+\zeta\right) .
$$

With the triangle inequality, (4.2), and the above inequality, we obtain

$$
\begin{aligned}
\bar{v}\left(\boldsymbol{y}_{\hat{\tau}_{n}}\right) & =\bar{v}\left(\boldsymbol{y}_{\hat{\tau}_{n}-}+\hat{\zeta}_{n}\right) \\
& \geq \bar{v}\left(\boldsymbol{y}_{\hat{\tau}_{n}-}+\hat{\zeta}_{n}+\zeta\right)+\bar{C}\left(\boldsymbol{y}_{\hat{\tau}_{n}-}, \hat{\zeta}_{n}+\zeta\right)-\bar{C}\left(\boldsymbol{y}_{\hat{\tau}_{n}-}, \hat{\zeta}_{n}\right)-\frac{\delta}{2^{n}} \\
& \geq \bar{v}\left(\boldsymbol{y}_{\hat{\tau}_{n}-}+\hat{\zeta}_{n}+\zeta\right)+\bar{C}\left(\boldsymbol{y}_{\hat{\tau}_{n}-}+\hat{\zeta}_{n}, \zeta\right)+\left|\log \left(1-c_{0}\right)\right|-\frac{\delta}{2^{n}} \\
& =\bar{v}\left(\boldsymbol{y}_{\hat{\tau}_{n}}+\zeta\right)+\bar{C}\left(\boldsymbol{y}_{\hat{\tau}_{n}}, \zeta\right)+\left|\log \left(1-c_{0}\right)\right|-\frac{\delta}{2^{n}} .
\end{aligned}
$$

Then $\bar{v}\left(\boldsymbol{y}_{\hat{\tau}_{n}}\right)>\mathcal{M} \bar{v}\left(\boldsymbol{y}_{\hat{\tau}_{n}}\right)$. This implies that $\hat{\tau}_{n}<\hat{\tau}_{n+1}$ a.s. 
From the definition of the strategy $\hat{a}_{d m}$ and Lemma 3.7, we obtain

$$
\begin{aligned}
J\left(\hat{a}_{d m}\right) & =r+\bar{l}+\liminf _{T \rightarrow \infty} \frac{1}{T} \mathrm{E}\left[\int_{0}^{T}\left(\bar{f}\left(\boldsymbol{y}_{s}\right)-\bar{l}\right) \mathrm{d} s+\sum_{n=1}^{\infty} \bar{C}\left(\boldsymbol{y}_{\hat{\tau}_{n+1}-}, \hat{\zeta}_{n+1}\right) 1_{\left\{\tau_{n}<T\right\}}\right] \\
& \geq r+\bar{l}+\liminf _{T \rightarrow \infty} \frac{1}{T} \mathrm{E}\left[\bar{v}(\boldsymbol{y})-\bar{v}\left(\boldsymbol{y}_{T}\right)-\sum_{n=1}^{\infty} \frac{\delta}{2^{n}}\right] \\
& =r+\bar{l} .
\end{aligned}
$$

This completes the proof.

\section{Acknowledgement}

I am very grateful to an anonymous referee for useful suggestions and comments.

\section{References}

[1] Akian, M., Sulem, A. And Taksar, M. I. (2001). Dynamic optimization of long-term growth rate for a portfolio with transaction costs and logarithmic utility. Math. Finance 11, 153-188.

[2] Bensoussan, A. (1982). Stochastic Control by Functional Analysis Methods (Studies Math. Appl. 11). NorthHolland, Amsterdam.

[3] Bensoussan, A. And Lions, J.-L. (1982). Applications of Variational Inequalities in Stochastic Control (Studies Math. Appl. 12). North-Holland, Amsterdam.

[4] Bensoussan, A. ANd Lions, J.-L. (1984). Impulse Control and Quasivariational Inequalities. Gauthier-Villars, Montrouge.

[5] Bielecki, T. R. and Pliska, S. R. (2000). Risk sensitive asset management with transaction costs. Finance Stoch. 4, 1-33.

[6] Davis, M. H. A. And Norman, A. R. (1990). Portfolio selection with transaction costs. Math. Operat. Res. 15, 676-713.

[7] Duffie, D. And Sun, T. S. (1990). Trans. costs and portfolio choice in a discrete-continuous-time setting. $J$. Econom. Dynamics Control 14, 35-51.

[8] Irle, A. AND SASs, J. (2006). Optimal portfolio policies under fixed and proportional transaction costs. Adv. Appl. Prob. 38, 916-942.

[9] Merton, R. C. (1969). Lifetime portfolio selection under uncertainty: the continuous-time case. Rev. Econom. Statist. 51, 247-257.

[10] Merton, R. C. (1971). Optimum consumption and portfolio rules in a continuous-time model. J. Econom. Theory 3, 373-413.

[11] Morton, A. And Pliska, S. R. (1995). Optimal portfolio management with fixed transaction costs. Math. Finance 5, 337-356.

[12] NagaI, H. (2004). Risky fraction processes and problems with transaction costs. In Stochastic Processes and Applications to Mathematical Finance, eds J. Akahori et al., World Scientific, River Edge, NJ, pp. 271-288.

[13] RobIN, M. (1981). On some impulse control problems with long run average cost. SIAM J. Control Optimization 19, 333-358.

[14] RobIn, M. (1983). Asymptotics in quasivariational inequalities and ergodic control problems. Systems Control Lett. 3, 171-176.

[15] Taksar, M., Klass, M. J. and Assaf, D. (1988). A diffusion model for optimal portfolio selection in the presence of brokerage fees. Math. Operat. Res. 13, 277-294.

[16] TAmura, T. (2006). Maximizing the growth rate of a portfolio with fixed and proportional transaction costs. Appl. Math. Optimization 54, 95-116. 